\title{
Search for young low-mass stars in a ROSAT selected sample south of the Taurus-Auriga molecular clouds ${ }^{\star, \star \star}$
}

\author{
A. Magazzù̀ ${ }^{1}$, E.L. Martín ${ }^{2}$, M.F. Sterzik ${ }^{3}$, R. Neuhäuser ${ }^{3}$, E. Covino ${ }^{4}$, and J.M. Alcalá ${ }^{3}$ \\ 1 Osservatorio Astrofisico di Catania, Città Universitaria, I-95125 Catania, Italy \\ 2 Instituto de Astrofísica de Canarias, E-38200 La Laguna, Tenerife, Spain \\ 3 Max-Planck-Institut für Extraterrestrische Physik, D-85740 Garching, Germany \\ 4 Osservatorio Astronomico di Capodimonte, Via Moiariello 16, I-80131 Napoli, Italy
}

Received July 29; accepted December 3, 1996

\begin{abstract}
We present results of intermediate resolution spectroscopy of 131 optical counterparts to 115 ROSAT All-Sky Survey X-ray sources south of the Taurus-Auriga dark cloud complex. These objects have been selected as candidate young stars from a total of 1084 ROSAT sources in a $\sim 300$ square degree area. We identify 30 objects as low-mass PMS stars on the basis of the Li $\lambda 6708 \AA$ doublet in their spectrum, a signature of their young age. All these stars have a spectral type later than F7 and show spectral characteristics typical of weak-line and post$\mathrm{T}$ Tauri stars. The presence of young objects several parsecs away from the regions of ongoing star formation is discussed in the light of the current models of $\mathrm{T}$ Tauri dispersal.
\end{abstract}

Key words: stars: formation - stars: late-type - stars: pre-main sequence $-\mathrm{X}$-rays: stars

\section{Introduction}

The study of T Tauri stars (TTS) is a main step towards the understanding of star formation in the Galaxy and early phases of low-mass stellar evolution. Early definitions (e.g. Herbig 1962; Bastian et al. 1983) describe TTS as low-mass pre-main sequence (PMS) objects showing in their spectrum emission from the hydrogen Balmer lines

Send offprint requests to: A. Magazzù

* Based on observations made with the Isaac Newton Telescope operated on the island of La Palma by the Royal Greenwich Observatory in the Spanish Observatorio del Roque de los Muchachos of the Instituto de Astrofísica de Canarias and with the ESO $1.52 \mathrm{~m}$ telescope on La Silla, Chile, operated by the European Southern Observatory.

$\star \star$ Tables 1, 2, 3, 4 are also available in electronic form at the CDS via anonymous ftp to cdsarc.u-strasbg.fr (130.79.128.5) or via http://cdsweb.u-strasbg.fr/Abstract.html and the $\mathrm{Ca}$ II $\mathrm{H}$ and $\mathrm{K}$ lines, reflecting the fact that TTS were discovered in $\mathrm{H} \alpha$ surveys of nearby molecular clouds ("classical" TTS, cTTS).

After the Einstein Observatory (EO) X-ray mission other objects have joined the T Tauri family, in particular X-ray active "weak-line" TTS (wTTS), which lack strong emission lines $\left(W_{\lambda}(\mathrm{H} \alpha) \leq 10 \AA\right.$ ) (e.g. Gahm 1980; Feigelson \& DeCampli 1981) and, usually, near-infrared excess emission (Walter 1986). From X-ray emission variability and the spatial incompletness of EO pointed observations, Walter et al. (1988) deduced that there should be as many as 1000 wTTS in the general Taurus-Auriga region, many of which can be discovered with the RASS. The population defined by the new wTTS discovered by Walter et al. (1988) by optical follow-up observations of previously unidentified EO sources cannot be identified with the post-TTS population proposed by Herbig (1978), as many wTTS share the same locus in the H-R diagram as cTTS. In the optical spectrum of all TTS the Li I $\lambda 6708 \AA$ absorption doublet is a prominent feature indicative of their PMS nature (Magazzù et al. 1992; Martín et al. 1994). The majority of TTS have been discovered in areas where molecular gas has been detected. One of the best studied areas is the Taurus-Auriga star forming region (SFR), a $\mathrm{T}$ association at a distance of $\sim 140 \mathrm{pc}$ (Elias 1978; Kenyon et al. 1994).

The advent of the ROSAT All-Sky Survey (RASS) has enabled us to extend the search for X-ray active low-mass stars to the complete sky, with a flux limit comparable with typical EO pointed observations. By studying X-ray spectra of RASS-detected well-known TTS and unidentified RASS sources in the Taurus-Auriga SFR, Neuhäuser et al. (1995a) have proposed that several hundreds of coronally active TTS are hidden in the RASS database. So far, 76 PMS stars have been discovered in the central parts of the Taurus-Auriga association (Wichmann et al. 1996). Some other PMS stars have been discovered with optical follow-up observations of sources found in deep ROSAT 
pointed observations in Taurus-Auriga (Strom \& Strom 1994; Carkner et al. 1996; Wichmann et al. 1996). Based on ROSAT observations, many new PMS stars have been discovered in other SFRs as well (see Alcalá et al. 1995; Neuhäuser 1996; Krautter 1996).

As the set of unidentified RASS sources is very large, Sterzik et al. (1995) proposed an efficient way of preselecting TTS candidates just from ROSAT and Hubble Space Telescope Guide Star Catalog (GSC) data alone, which are easily accessible for all unidentified RASS sources having a near-by GSC counterpart. Surprisingly, Sterzik et al. (1995) did not find any gradient in the space density of TTS candidates at the edges of the Orion molecular clouds and they proposed to extend the search for TTS even outside the cloud complexes. The strength of RASS as being spatially unbiased allows us to survey for TTS also outside the regions previously known to be populated by TTS.

In this paper, we report on the results of such a study conducted south of the Taurus-Auriga clouds. In Sect. 2, we describe ROSAT observations and X-ray data analysis. The method introduced by Sterzik et al. (1995) has been then applied to select TTS candidates (Sect. 3). Optical follow-up observations of these TTS candidates and data reduction are explained in Sect. 4, results are presented in Sect. 5 and discussed in the last section.

In addition to data presented here, high-resolution spectral observations have been obtained for most of our stars to study their radial velocity; these data as well as proper motions of several new PMS stars are given in Neuhäuser et al. (1997), in which the kinematics and possible modes of origin of newly discovered PMS stars are discussed. Preliminary results of our study were reported by Neuhäuser et al. (1995c), who discussed the results of optical observations of 15 RASS-selected TTS candidates south of Taurus-Auriga. These objects are included in the sample discussed here.

\section{X-ray data}

The X-ray telescope ROSAT and its detectors are described in detail by Trümper (1983) and Pfeffermann et al. (1988). The Position Sensitive Proportional Counter (PSPC) on board ROSAT performed an All-Sky Survey scanning the sky in great circles with a $2^{\circ}$ diameter field of view. Observations in our selected area have been performed in August and September 1990 and in February 1991. Vignetting corrected RASS exposure times vary roughly with $1 / \cos \beta$ where $\beta$ denotes ecliptic latitude. In our studied area, the exposure varies between $\sim 430$ and $\sim 580$ seconds. During the RASS, all areas in the sky have been observed in $\sim 30$ second long scans, separated by $\sim 90$ minutes and spread over almost two days. The ROSAT PSPC has 255 instrumental energy channels sensitive from $0.1 \mathrm{keV}$ to $2.4 \mathrm{keV}$ (broad band). The spectral resolution of the ROSAT PSPC instrument
$43 \%$ at $0.93 \mathrm{keV})$ permits a reasonable spectral analysis in three energy bands:

- soft: 0.1 to $0.4 \mathrm{keV}$

- hard 1: 0.5 to $0.9 \mathrm{keV}$

- hard 2: 0.9 to $2.1 \mathrm{keV}$.

In the flux-limited RASS, most of the previously known wTTS but only few cTTS have been detected (Neuhäuser et al. 1995b). It has been shown (Neuhäuser et al. 1995a,b) that wTTS and cTTS can be discriminated by their X-ray spectral hardness ratios. If $Z_{\mathrm{s}}, Z_{\mathrm{h} 1}$, and $Z_{\mathrm{h} 2}$ denote count rates for the ROSAT energy channels soft, hard 1, and hard 2, respectively, we define two hardness ratios as follows:

$H R 1=\frac{Z_{\mathrm{h} 1}+Z_{\mathrm{h} 2}-Z_{\mathrm{s}}}{Z_{\mathrm{h} 1}+Z_{\mathrm{h} 2}+Z_{\mathrm{s}}} ; \quad H R 2=\frac{Z_{\mathrm{h} 2}-Z_{\mathrm{h} 1}}{Z_{\mathrm{h} 2}+Z_{\mathrm{h} 1}}$.

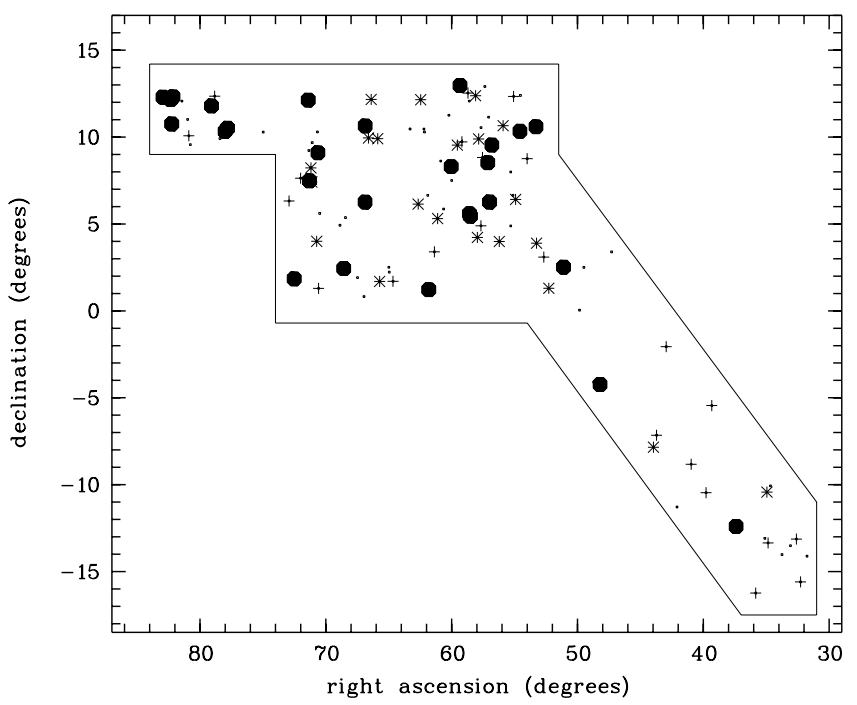

Fig. 1. Taurus-Auriga: In our studied area (enclosed by a box) we find new PMS stars (filled symbols), possible new PMS stars (stars), new dKe/dMe stars (plusses) and other non-PMS stars (dots)

We have extracted from the merged photon event, exposure, and attitude files of the RASS data those data which pertain to our studied area south of the TaurusAuriga dark clouds. This area is shown in Fig. 1; it includes a region just south of the Taurus-Auriga dark cloud complex (i.e. with $\delta \leq 14^{\circ}$ ) and an attached strip perpendicular to the galactic plane. Neuhäuser et al. (1995c) refer to the same area, while the region north of $\delta=14^{\circ}$ has been searched for new TTS by Wichmann et al. (1996). Source detection and local background determination were done separately in five different ROSAT energy bands: broad, soft, hard 1, hard 2, and hard (0.5 to $2.1 \mathrm{keV})$. Detected sources were merged and tested again with a maximum likelihood technique. Details on RASS data reduction and source detection algorithm can be found in 
Neuhäuser et al. (1995b). These authors showed that all sources with a maximum likelihood of existence of at least 7.4 can be accepted as real sources; such a low likelihood value is justified as we search for unknown TTS which may be X-ray faint.

\section{Source selection}

Out of the 1084 RASS sources in our studied region, only 179 (about 17\%) can be identified with known stellar and extragalactic counterparts in the Simbad database. Clearly, for efficient ground-based optical follow-up observations a pre-selection among the remaining sample of more than $900 \mathrm{X}$-ray sources is needed. A general statistical discrimination method was introduced by Sterzik et al. (1995) in order to find promising TTS candidates in the ROSAT database and was applied to study the spatial distribution of TTS candidates in the Orion SFR. In the present case, we apply the same method to our sample of RASS sources. A detailed description of the procedure can be found in Sterzik et al. (1995). Here we only summarize the relevant steps:

1. We establish a "training set", consisting of members of previously classified groups, in our case TTS and non-TTS. As our observations are carried out close to the Taurus SFR, we conveniently draw the sources of the training set from follow-up observations of ROSAT sources reported by Wichmann et al. (1996). We use the properties of 63 TTS and 87 non-TTS of their survey (all detected in the RASS). We added also 54 RASS-detected TTS from the Herbig \& Bell (1988) catalog. Our training set presents a uniform spatial distribution.

2. As discrimination parameters we choose: $H R 1$ and $H R 2$ determined for each X-ray source, $V$ of the closest optical counterpart in the GSC within $40^{\prime \prime}$ around the X-ray source, and the X-ray to optical flux ratio $f_{\mathrm{X}} / f_{V}$. If no GSC counterpart is present within $40^{\prime \prime}$ we assign $V=20$, indicating a faint optical X-ray source not detected in the GSC.

3. A $k$-nearest neighbourhood analysis is applied to all sources in the sample. A discrimination probability $P_{i}$ for each source $i$ is defined as

$P_{i}=\frac{n(\mathrm{TTS})}{k}$,

where $n$ (TTS) is the number of TTS in the training set "in the neighbourhood" of source $i$ in the four-dimensional discrimination parameter space. The neighbourhood is defined as the sphere containing exactly

$n(\mathrm{TTS})+n($ non-TTS $)=k$

members of the training set. For the present study we have chosen $k=16$, because this value proved to give the best results in telling TTS from non-TTS within the training set. High values of $P_{i}$ indicate that the source properties are similar to those in the sample of TTS of the training set.

Source selection on this basis implies a bias towards (X-ray and optical) properties of typical TTS in the training set, as desired. However, the selection cannot guarantee a "pure" TTS sample, because other classes of stars such as active binaries, RS CVn binaries, emission line dwarfs, etc., share similar values within the discrimination parameter set, and are expected to contaminate any sub-sample.

Figure 2 summarizes the definition of the X-ray sample according to the discrimination probability $P$. We note that, above a discrimination threshold of $0.6(0.5)$, we have observed 97 (110) sources out of a total of 180 (246) candidates. It can be seen that many X-ray sources with high discrimination probability, i.e. many TTS candidates, indeed are new PMS stars (see Sects. 4, 5), while there are no new PMS stars among X-ray sources with low discrimination probability.

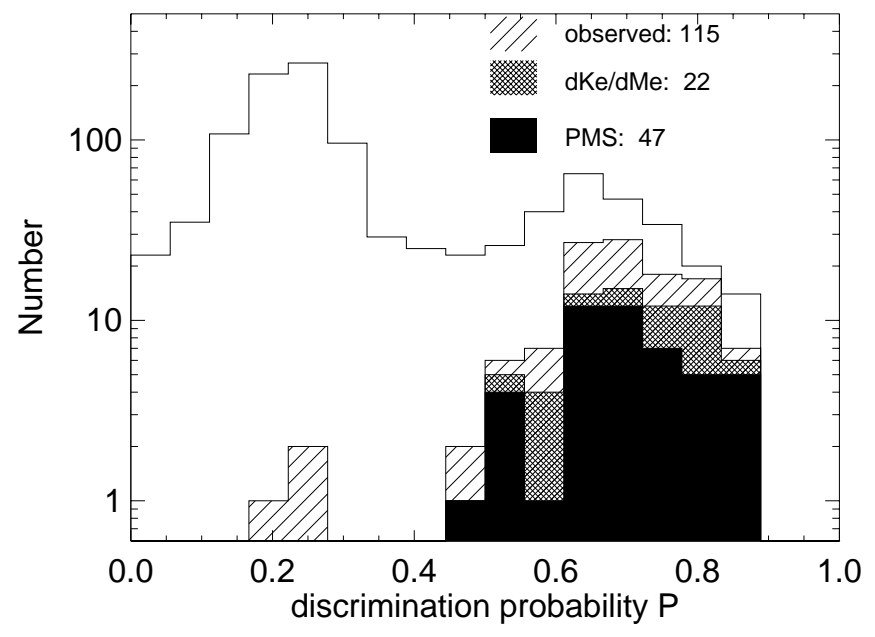

Fig. 2. Source selection: The histogram gives the distribution of discrimination probability $P$ for all $1084 \mathrm{X}$-ray sources in our studied area. Plotted is the number of stars per $P$ bin (with bin size 0.05 ). The hatched histogram indicates the observed sub-sample with dKe/dMe stars and new PMS stars (including certain and possible new PMS stars). There are a total of 47 X-ray counterparts classified as either PMS or PMS? and 22 other X-ray counterparts classified as dKe or dMe stars. There are no RASS sources with $P>0.9$

Whereas previously this classification scheme was primarily applied to demonstrate the discrimination ability within the training set itself (as the only a priori known classified sample), the results of this work indeed prove the prediction quality of the suggested procedure and allow a reliable extrapolation of the number of different types of active stars in the sample.

In Table 1 we list all the RASS sources selected in the way described above. We list ROSAT source name, 
Table 1. X-ray data for our sample. Listed are all X-ray sources detected in the ROSAT All-Sky Survey (sorted by right ascension), studied optically in this work. We list ROSAT source designation (X-ray positions can be obtained from data in Table 2), number of counts in the broad ROSAT band with errors, exposure time, hardness ratios with errors, the maximum likelihood of existence $M L$, the emission energy (typical error being $\pm 0.55 \mathrm{keV}$ ), the absorbing foreground column density (typical error \pm 0.77 , in $\log \left(N_{\mathrm{H}}\right) \mathrm{cm}^{-2}$ ), and finally the X-ray flux (typical error $\pm 1.8710^{-13} \mathrm{erg} \mathrm{s}^{-1} \mathrm{~cm}^{-2}$ )

\begin{tabular}{|c|c|c|c|c|c|c|c|c|}
\hline designation & counts & $\begin{array}{c}\exp \\
(\mathrm{s})\end{array}$ & $H R 1$ & $H R 2$ & $M L$ & $\begin{array}{c}k T_{\mathrm{X}} \\
(\mathrm{keV})\end{array}$ & $\begin{array}{c}\log \left(N_{\mathrm{H}}\right) \\
\left(\mathrm{cm}^{-2}\right)\end{array}$ & $\begin{array}{c}f_{\mathrm{X}} / 10^{-13} \\
\left(\mathrm{erg} \mathrm{s}^{-1} \mathrm{~cm}^{-2}\right)\end{array}$ \\
\hline RXJ 0207.0-1407 & $7.1 \pm 3.3$ & 442.1 & $1.00 \pm 0.21$ & $0.69 \pm 0.31$ & 14.3 & 1.14 & 22.00 & 19.56 \\
\hline RXJ 0209.1-1536 & $16.3 \pm 5.5$ & 444.2 & $0.95 \pm 0.49$ & $-0.09 \pm 0.35$ & 21.8 & 0.72 & 20.85 & 5.17 \\
\hline RXJ 0210.4-1308 & $21.5 \pm 7.3$ & 548.2 & $0.35 \pm 0.31$ & $0.20 \pm 0.39$ & 17.7 & 1.07 & 18.10 & 3.53 \\
\hline RXJ 0212.3-1330 & $17.9 \pm 6.8$ & 556.2 & $0.20 \pm 0.38$ & $0.23 \pm 0.45$ & 10.9 & 1.22 & 18.00 & 2.98 \\
\hline RXJ 0215.0-1402 & $9.9 \pm 4.5$ & 529.3 & $0.67 \pm 0.38$ & $0.43 \pm 0.53$ & 12.4 & 1.67 & 20.35 & 3.06 \\
\hline RXJ 0218.6-1004 & $17.9 \pm 5.6$ & 486.2 & $1.00 \pm 0.12$ & $-0.04 \pm 0.31$ & 21.7 & 0.58 & 21.55 & 11.53 \\
\hline RXJ 0219.4-1321 & $11.6 \pm 4.7$ & 523.8 & $0.92 \pm 0.63$ & $-0.03 \pm 0.40$ & 12.3 & 0.81 & 20.60 & 2.76 \\
\hline RXJ 0219.7-1026 & $46.2 \pm 9.4$ & 530.5 & $0.49 \pm 0.19$ & $0.46 \pm 0.19$ & 53.1 & 1.70 & 20.10 & 12.16 \\
\hline RXJ 0220.4-1305 & $15.8 \pm 6.5$ & 506.5 & $0.07 \pm 0.45$ & $-0.23 \pm 0.25$ & 10.5 & 0.39 & 18.00 & 2.31 \\
\hline RXJ 0223.3-1615 & $8.6 \pm 4.1$ & 532.1 & $1.00 \pm 0.22$ & $0.18 \pm 0.46$ & 9.6 & 0.57 & 21.85 & 21.09 \\
\hline RXJ 0229.5-1224 & $12.3 \pm 5.0$ & 516.4 & $0.60 \pm 0.35$ & $0.41 \pm 0.37$ & 12.8 & 1.68 & 20.25 & 3.70 \\
\hline RXJ 0237.3-0527 & $9.2 \pm 4.5$ & 279.4 & $0.22 \pm 0.48$ & $0.44 \pm 0.68$ & 7.7 & 1.63 & 19.45 & 3.60 \\
\hline RXJ 0239.1-1028 & $6.5 \pm 3.3$ & 326.0 & $0.33 \pm 0.51$ & $0.26 \pm 0.24$ & 12.7 & 1.15 & 18.90 & 1.91 \\
\hline RXJ 0243.9-0850 & $8.3 \pm 4.5$ & 341.7 & $0.44 \pm 0.44$ & $0.55 \pm 0.74$ & 7.5 & 1.67 & 20.00 & 3.22 \\
\hline RXJ 0248.3-1117 & $8.0 \pm 4.3$ & 418.7 & $0.16 \pm 0.28$ & $0.01 \pm 0.38$ & 7.7 & 1.15 & 18.00 & 1.76 \\
\hline RXJ 0251.8-0203 & $8.5 \pm 3.9$ & 325.7 & $0.43 \pm 0.43$ & $0.09 \pm 0.46$ & 12.6 & 0.96 & 18.00 & 2.28 \\
\hline RXJ 0254.8-0709 & $10.4 \pm 4.4$ & 329.7 & $0.70 \pm 0.32$ & $0.76 \pm 0.26$ & 15.7 & 1.94 & 21.80 & 20.03 \\
\hline RXJ 0255.8-0750 & $8.7 \pm 4.3$ & 330.5 & $0.89 \pm 0.80$ & $0.30 \pm 0.42$ & 10.0 & 1.17 & 20.60 & 4.15 \\
\hline RXJ 0309.1+0324 & $28.5 \pm 7.2$ & 614.3 & $0.81 \pm 0.19$ & $0.39 \pm 0.24$ & 38.1 & 1.39 & 20.50 & 7.75 \\
\hline RXJ 0312.8-0414 & $34.0 \pm 7.9$ & 424.7 & $0.64 \pm 0.20$ & $0.07 \pm 0.23$ & 43.4 & 0.93 & 19.70 & 8.31 \\
\hline RXJ 0314.8-0406 & $6.7 \pm 3.9$ & 465.8 & $1.00 \pm 0.29$ & $0.46 \pm 0.48$ & 8.5 & 0.76 & 22.00 & 24.47 \\
\hline RXJ $0317.9+0231$ & $14.3 \pm 5.6$ & 541.9 & $1.00 \pm 0.16$ & $-0.01 \pm 0.39$ & 12.2 & 0.58 & 21.60 & 10.67 \\
\hline RXJ 0319.3+0003 & $8.7 \pm 4.4$ & 603.2 & $1.00 \pm 0.26$ & $-0.10 \pm 0.51$ & 8.8 & 0.55 & 21.45 & 4.66 \\
\hline RXJ 0324.4+0231 & $21.7 \pm 7.1$ & 461.1 & $0.36 \pm 0.34$ & $0.15 \pm 0.38$ & 16.6 & 1.03 & 18.00 & 4.23 \\
\hline RXJ 0329.1+0118 & $11.9 \pm 4.9$ & 637.8 & $1.00 \pm 0.14$ & $0.50 \pm 0.35$ & 12.5 & 0.83 & 22.00 & 28.73 \\
\hline RXJ $0330.7+0306$ & $54.4 \pm 9.8$ & 737.7 & $1.00 \pm 0.04$ & $0.05 \pm 0.18$ & 54.9 & 0.59 & 21.70 & 40.64 \\
\hline RXJ $0333.0+0354$ & $9.9 \pm 4.5$ & 634.2 & $1.00 \pm 0.22$ & $0.57 \pm 0.46$ & 10.5 & 0.94 & 22.00 & 21.98 \\
\hline RXJ $0333.1+1036$ & $20.3 \pm 6.2$ & 564.3 & $0.36 \pm 0.25$ & $0.48 \pm 0.26$ & 30.8 & 1.66 & 19.85 & 4.55 \\
\hline RXJ 0336.0+0846 & $8.7 \pm 4.0$ & 543.0 & $0.78 \pm 0.85$ & $-0.26 \pm 0.44$ & 10.7 & 0.51 & 20.30 & 1.72 \\
\hline RXJ $0338.1+1224$ & $8.3 \pm 4.2$ & 555.1 & $1.00 \pm 0.23$ & $0.25 \pm 0.50$ & 10.0 & 0.60 & 21.90 & 18.40 \\
\hline RXJ 0338.3+1020 & $13.2 \pm 4.8$ & 529.8 & $1.00 \pm 0.16$ & $0.17 \pm 0.35$ & 15.1 & 0.64 & 21.80 & 19.04 \\
\hline RXJ 0339.6+0624 & $8.6 \pm 4.1$ & 569.3 & $1.00 \pm 0.20$ & $0.47 \pm 0.40$ & 9.7 & 0.78 & 22.00 & 24.42 \\
\hline RXJ $0340.3+1220$ & $26.9 \pm 6.6$ & 517.6 & $0.85 \pm 0.17$ & $-0.18 \pm 0.25$ & 49.3 & 0.64 & 20.35 & 5.77 \\
\hline RXJ 0340.5+0639 & $5.3 \pm 2.9$ & 522.8 & $1.00 \pm 0.26$ & $0.26 \pm 0.25$ & 9.8 & 0.61 & 21.90 & 12.48 \\
\hline RXJ $0341.2+0453$ & $37.6 \pm 8.2$ & 538.1 & $1.00 \pm 0.04$ & $0.26 \pm 0.21$ & 56.6 & 0.63 & 21.90 & 81.67 \\
\hline RXJ $0341.2+0759$ & $8.1 \pm 3.9$ & 567.9 & $1.00 \pm 0.23$ & $0.19 \pm 0.47$ & 8.3 & 0.59 & 21.85 & 17.56 \\
\hline RXJ 0343.6+1039 & $17.5 \pm 6.0$ & 555.3 & $0.89 \pm 0.30$ & $0.60 \pm 0.24$ & 21.0 & 2.02 & 21.45 & 11.78 \\
\hline RXJ $0344.8+0359$ & $25.5 \pm 6.9$ & 529.7 & $0.88 \pm 0.13$ & $-0.15 \pm 0.29$ & 29.0 & 0.67 & 20.45 & 5.55 \\
\hline RXJ 0347.2+0933 & $16.1 \pm 5.4$ & 527.6 & $0.58 \pm 0.28$ & $0.85 \pm 0.16$ & 19.9 & 2.02 & 22.00 & 32.54 \\
\hline RXJ 0347.9+0616 & $8.6 \pm 4.0$ & 466.0 & $1.00 \pm 0.24$ & $0.43 \pm 0.19$ & 11.3 & 0.70 & 22.00 & 33.14 \\
\hline RXJ 0348.2+1109 & $23.8 \pm 6.5$ & 471.2 & $0.30 \pm 0.28$ & $-0.07 \pm 0.32$ & 29.7 & 0.93 & 18.00 & 4.41 \\
\hline RXJ $0348.5+0832$ & $23.3 \pm 6.4$ & 486.9 & $0.92 \pm 0.23$ & $-0.28 \pm 0.29$ & 30.6 & 0.44 & 20.80 & 6.81 \\
\hline RXJ 0349.4+1255 & $19.7 \pm 5.7$ & 479.3 & $0.81 \pm 0.35$ & $-0.25 \pm 0.29$ & 42.5 & 0.53 & 20.35 & 4.56 \\
\hline RXJ $0350.2+0849$ & $6.6 \pm 3.3$ & 470.2 & $1.00 \pm 0.24$ & $0.07 \pm 0.51$ & 11.8 & 0.61 & 21.70 & 7.74 \\
\hline RXJ 0350.6+0454 & $7.4 \pm 3.7$ & 488.1 & $1.00 \pm 0.23$ & $0.09 \pm 0.50$ & 11.6 & 0.58 & 21.75 & 12.09 \\
\hline RXJ $0350.6+1033$ & $12.5 \pm 5.3$ & 569.5 & $1.00 \pm 0.17$ & $0.51 \pm 0.33$ & 9.4 & 0.85 & 22.00 & 33.80 \\
\hline RXJ $0351.4+0953$ & $11.8 \pm 4.9$ & 491.4 & $0.90 \pm 0.24$ & $-0.25 \pm 0.42$ & 8.8 & 0.51 & 20.65 & 2.95 \\
\hline RXJ $0351.8+0413$ & $6.5 \pm 3.3$ & 441.1 & $1.00 \pm 0.25$ & $-0.10 \pm 0.52$ & 9.2 & 0.55 & 21.45 & 4.77 \\
\hline RXJ $0352.4+1223$ & $11.8 \pm 4.8$ & 522.4 & $1.00 \pm 0.18$ & $-0.00 \pm 0.42$ & 13.3 & 0.55 & 21.65 & 9.48 \\
\hline RXJ $0354.1+0528$ & $7.4 \pm 4.1$ & 535.0 & $1.00 \pm 0.26$ & $0.55 \pm 0.19$ & 7.6 & 0.91 & 22.00 & 20.34 \\
\hline
\end{tabular}


Table 1. continued

\begin{tabular}{|c|c|c|c|c|c|c|c|c|}
\hline designation & counts & $\begin{array}{c}\exp \\
(\mathrm{s})\end{array}$ & $H R 1$ & $H R 2$ & $M L$ & $\begin{array}{c}k T_{\mathrm{X}} \\
(\mathrm{keV})\end{array}$ & $\begin{array}{c}\log \left(N_{\mathrm{H}}\right) \\
\left(\mathrm{cm}^{-2}\right)\end{array}$ & $\begin{array}{c}f_{\mathrm{X}} / 10^{-13} \\
\left(\mathrm{erg} \mathrm{s}^{-1} \mathrm{~cm}^{-2}\right)\end{array}$ \\
\hline RXJ $0354.3+0535$ & $19.2 \pm 6.1$ & 536.7 & $1.00 \pm 0.11$ & $0.42 \pm 0.31$ & 24.7 & 0.69 & 22.00 & 64.23 \\
\hline RXJ 0354.4+1204 & $8.2 \pm 3.9$ & 474.2 & $0.58 \pm 0.39$ & $1.00 \pm 1.00$ & 13.2 & 1.62 & 20.20 & 2.53 \\
\hline RXJ $0354.8+1232$ & $16.6 \pm 5.9$ & 505.8 & $0.81 \pm 0.33$ & $-0.11 \pm 0.36$ & 18.3 & 0.73 & 20.15 & 3.42 \\
\hline RXJ 0356.7+0943 & $10.3 \pm 5.1$ & 507.5 & $0.60 \pm 0.39$ & $-0.40 \pm 0.42$ & 9.1 & 0.32 & 20.35 & 2.80 \\
\hline RXJ $0357.3+1258$ & $17.9 \pm 6.1$ & 486.0 & $0.65 \pm 0.24$ & $-0.39 \pm 0.30$ & 15.9 & 0.33 & 20.40 & 4.63 \\
\hline RXJ 0358.1+0932 & $27.6 \pm 6.9$ & 467.6 & $0.86 \pm 0.22$ & $0.57 \pm 0.20$ & 42.7 & 2.02 & 21.35 & 19.43 \\
\hline RXJ 0400.0+0730 & $10.0 \pm 4.1$ & 459.9 & $1.00 \pm 0.15$ & $0.61 \pm 0.33$ & 14.9 & 1.00 & 22.00 & 29.38 \\
\hline RXJ 0400.1+0818 & $169.6 \pm 16.6$ & 450.7 & $0.27 \pm 0.10$ & $0.17 \pm 0.12$ & 298.5 & 1.11 & 18.00 & 34.22 \\
\hline RXJ 0400.8+1116 & $15.7 \pm 5.7$ & 494.8 & $1.00 \pm 0.15$ & $-0.05 \pm 0.38$ & 13.4 & 0.56 & 21.55 & 10.26 \\
\hline RXJ 0402.5+0552 & $18.2 \pm 5.8$ & 414.1 & $0.68 \pm 0.27$ & $0.24 \pm 0.37$ & 19.5 & 1.11 & 20.05 & 5.37 \\
\hline RXJ 0403.5+0837 & $20.2 \pm 6.2$ & 480.6 & $1.00 \pm 0.09$ & $0.56 \pm 0.25$ & 22.3 & 0.93 & 22.00 & 59.17 \\
\hline RXJ 0404.4+0519 & $20.3 \pm 5.6$ & 413.6 & $1.00 \pm 0.06$ & $-0.06 \pm 0.28$ & 41.8 & 0.57 & 21.55 & 15.87 \\
\hline RXJ 0405.5+0324 & $34.2 \pm 7.8$ & 445.4 & $1.00 \pm 0.04$ & $0.03 \pm 0.23$ & 54.4 & 0.57 & 21.70 & 44.18 \\
\hline RXJ $0407.2+0113$ & $10.1 \pm 4.1$ & 421.0 & $1.00 \pm 0.16$ & $0.35 \pm 0.39$ & 13.0 & 0.52 & 22.00 & 56.03 \\
\hline RXJ 0407.6+0638 & $19.2 \pm 6.4$ & 474.5 & $1.00 \pm 0.08$ & $-0.25 \pm 0.34$ & 16.3 & 0.46 & 21.30 & 9.69 \\
\hline RXJ 0408.6+1017 & $5.9 \pm 3.4$ & 375.6 & $1.00 \pm 0.25$ & $0.53 \pm 0.43$ & 8.0 & 0.88 & 22.00 & 23.10 \\
\hline RXJ 0408.8+1028 & $106.0 \pm 13.6$ & 387.9 & $0.52 \pm 0.11$ & $0.24 \pm 0.14$ & 159.7 & 1.11 & 19.65 & 29.51 \\
\hline RXJ 0409.8+1209 & $10.5 \pm 5.1$ & 357.3 & $0.76 \pm 0.65$ & $0.30 \pm 0.23$ & 7.7 & 1.19 & 20.30 & 4.21 \\
\hline RXJ 0410.6+0608 & $8.7 \pm 3.9$ & 395.1 & $1.00 \pm 0.19$ & $0.08 \pm 0.46$ & 10.1 & 0.56 & 21.75 & 18.47 \\
\hline RXJ 0413.2+1028 & $13.0 \pm 4.6$ & 374.5 & $1.00 \pm 0.01$ & $0.56 \pm 0.11$ & 20.0 & 0.97 & 22.00 & 48.87 \\
\hline RXJ 0418.6+0143 & $9.2 \pm 4.5$ & 411.3 & $0.52 \pm 0.40$ & $0.46 \pm 0.39$ & 8.2 & 1.61 & 20.10 & 3.11 \\
\hline RXJ 0419.8+0214 & $17.3 \pm 6.1$ & 404.5 & $1.00 \pm 0.12$ & $0.11 \pm 0.35$ & 13.6 & 0.62 & 21.75 & 34.11 \\
\hline RXJ 0419.9+0231 & $47.7 \pm 9.7$ & 385.4 & $1.00 \pm 0.04$ & $0.06 \pm 0.18$ & 12.5 & 0.61 & 21.70 & 7.69 \\
\hline RXJ 0422.9+0141 & $15.4 \pm 5.9$ & 444.8 & $0.90 \pm 0.25$ & $0.50 \pm 0.33$ & 10.1 & 1.78 & 20.85 & 7.51 \\
\hline RXJ 0423.5+0955 & $33.9 \pm 8.8$ & 577.2 & $0.01 \pm 0.26$ & $-0.00 \pm 0.35$ & 22.9 & 2.02 & 18.00 & 5.54 \\
\hline RXJ 0425.5+1210 & $21.3 \pm 6.2$ & 641.0 & $1.00 \pm 0.08$ & $-0.09 \pm 0.29$ & 23.5 & 0.55 & 21.50 & 10.75 \\
\hline RXJ 0426.4+0957 & $16.3 \pm 5.6$ & 657.7 & $1.00 \pm 0.12$ & $-0.09 \pm 0.34$ & 18.6 & 0.54 & 21.50 & 8.01 \\
\hline RXJ 0427.4+1039 & $20.6 \pm 6.3$ & 654.3 & $1.00 \pm 0.10$ & $0.62 \pm 0.25$ & 23.3 & 1.02 & 22.00 & 42.55 \\
\hline RXJ 0427.5+0616 & $67.2 \pm 11.4$ & 612.2 & $0.37 \pm 0.16$ & $-0.06 \pm 0.20$ & 75.5 & 0.90 & 18.00 & 9.45 \\
\hline RXJ 0427.8+0049 & $17.8 \pm 6.0$ & 495.9 & $0.84 \pm 0.23$ & $-0.05 \pm 0.34$ & 17.7 & 0.80 & 20.25 & 4.04 \\
\hline RXJ 0429.9+0155 & $15.1 \pm 5.1$ & 534.7 & $1.00 \pm 0.11$ & $0.33 \pm 0.30$ & 18.5 & 0.63 & 21.95 & 53.69 \\
\hline RXJ $0433.7+0522$ & $15.1 \pm 6.3$ & 817.3 & $1.00 \pm 0.18$ & $0.31 \pm 0.39$ & 10.2 & 0.59 & 21.95 & 37.34 \\
\hline RXJ $0434.3+0226$ & $33.6 \pm 8.1$ & 775.6 & $1.00 \pm 0.04$ & $0.11 \pm 0.24$ & 39.0 & 0.56 & 21.80 & 36.33 \\
\hline RXJ $0435.5+0455$ & $19.6 \pm 6.0$ & 746.2 & $1.00 \pm 0.08$ & $0.37 \pm 0.26$ & 25.4 & 0.58 & 22.00 & 53.09 \\
\hline RXJ 0441.9+0537 & $96.3 \pm 13.6$ & 582.9 & $1.00 \pm 0.01$ & $0.50 \pm 0.12$ & 123.1 & 0.89 & 22.00 & 242.90 \\
\hline RXJ 0442.3+0118 & $19.8 \pm 7.1$ & 528.9 & $0.27 \pm 0.44$ & $0.34 \pm 0.43$ & 14.1 & 1.31 & 19.15 & 3.76 \\
\hline RXJ 0442.5+0906 & $22.0 \pm 6.4$ & 532.0 & $0.36 \pm 0.28$ & $-0.03 \pm 0.29$ & 24.2 & 0.91 & 18.00 & 3.56 \\
\hline RXJ 0442.6+1018 & $83.9 \pm 12.0$ & 537.8 & $0.90 \pm 0.07$ & $0.07 \pm 0.14$ & 142.0 & 0.91 & 20.55 & 19.83 \\
\hline RXJ 0442.9+0400 & $17.4 \pm 5.9$ & 532.6 & $1.00 \pm 0.11$ & $0.11 \pm 0.34$ & 21.3 & 0.62 & 21.75 & 26.06 \\
\hline RXJ 0444.3+0941 & $62.2 \pm 10.4$ & 568.1 & $0.58 \pm 0.14$ & $0.11 \pm 0.17$ & 95.5 & 0.97 & 19.55 & 10.84 \\
\hline RXJ 0444.4+0725 & $12.6 \pm 4.9$ & 514.6 & $1.00 \pm 0.15$ & $-0.30 \pm 0.39$ & 12.6 & 0.35 & 21.30 & 7.16 \\
\hline RXJ $0444.7+0814$ & $18.9 \pm 6.6$ & 571.0 & $0.58 \pm 0.33$ & $0.32 \pm 0.35$ & 19.2 & 1.25 & 20.00 & 4.27 \\
\hline RXJ $0445.2+0729$ & $10.1 \pm 5.0$ & 568.8 & $1.00 \pm 0.21$ & $0.19 \pm 0.46$ & 8.0 & 0.59 & 21.85 & 21.86 \\
\hline RXJ 0445.3+0914 & $9.5 \pm 4.7$ & 600.6 & $1.00 \pm 0.21$ & $0.43 \pm 0.51$ & 8.9 & 0.70 & 22.00 & 28.40 \\
\hline RXJ 0445.5+1207 & $9.4 \pm 4.4$ & 617.3 & $0.78 \pm 0.50$ & $0.60 \pm 0.40$ & 11.6 & 2.02 & 21.40 & 5.01 \\
\hline RXJ 0448.0+0738 & $88.8 \pm 12.8$ & 535.0 & $0.28 \pm 0.14$ & $-0.13 \pm 0.18$ & 93.9 & 0.44 & 18.00 & 12.52 \\
\hline RXJ 0450.0+0151 & $20.6 \pm 6.3$ & 535.6 & $0.95 \pm 0.21$ & $-0.08 \pm 0.31$ & 21.1 & 0.74 & 20.80 & 5.16 \\
\hline RXJ 0451.6+0619 & $9.5 \pm 4.3$ & 558.8 & $1.00 \pm 0.18$ & $-0.04 \pm 0.45$ & 8.9 & 0.58 & 21.55 & 5.33 \\
\hline RXJ 0459.9+1017 & $29.4 \pm 7.7$ & 603.5 & $1.00 \pm 0.05$ & $0.32 \pm 0.23$ & 27.8 & 0.62 & 21.95 & 98.47 \\
\hline RXJ 0511.2+1031 & $18.4 \pm 6.4$ & 661.5 & $1.00 \pm 0.13$ & $0.26 \pm 0.37$ & 15.7 & 0.62 & 21.90 & 34.24 \\
\hline RXJ 0511.9+1112 & $12.9 \pm 4.8$ & 618.1 & $0.71 \pm 0.36$ & $0.29 \pm 0.37$ & 12.8 & 1.18 & 20.20 & 2.87 \\
\hline RXJ 0512.0+1020 & $23.6 \pm 6.7$ & 651.2 & $0.78 \pm 0.21$ & $0.13 \pm 0.29$ & 23.4 & 0.99 & 20.20 & 4.46 \\
\hline RXJ 0513.6+0955 & $9.2 \pm 3.9$ & 593.2 & $0.43 \pm 0.42$ & $-0.45 \pm 0.17$ & 14.4 & 0.29 & 20.25 & 1.98 \\
\hline
\end{tabular}


Table 1. continued

\begin{tabular}{lrrrrrrrr}
\hline designation & counts & \multicolumn{1}{c}{$\begin{array}{c}\text { exp } \\
\text { (s) }\end{array}$} & HR1 & HR2 & $M L$ & $\begin{array}{c}k T_{\mathrm{X}} \\
(\mathrm{keV})\end{array}$ & $\begin{array}{r}\log \left(N_{\mathrm{H}}\right) \\
\left(\mathrm{cm}^{-2}\right)\end{array}$ & $\begin{array}{r}f_{\mathrm{X}} / 10^{-13} \\
\left(\mathrm{erg} \mathrm{s}^{-1} \mathrm{~cm}^{-2}\right)\end{array}$ \\
\hline RXJ 0515.3+1221 & $27.5 \pm 7.9$ & 690.5 & $0.57 \pm 0.24$ & $-0.07 \pm 0.30$ & 24.4 & 0.79 & 19.05 & 3.46 \\
RXJ 0516.3+1148 & $24.9 \pm 7.3$ & 658.6 & $0.98 \pm 0.24$ & $-0.03 \pm 0.29$ & 23.8 & 0.54 & 21.60 & 15.87 \\
RXJ 0523.0+0934 & $8.3 \pm 4.1$ & 429.6 & $0.74 \pm 0.52$ & $-0.25 \pm 0.51$ & 8.0 & 0.54 & 20.15 & 1.92 \\
RXJ 0523.5+1005 & $28.7 \pm 6.9$ & 466.5 & $0.93 \pm 0.15$ & $0.15 \pm 0.22$ & 49.7 & 0.98 & 20.70 & 9.10 \\
RXJ 0523.9+1101 & $6.8 \pm 3.3$ & 498.8 & $0.28 \pm 0.40$ & $-0.10 \pm 0.24$ & 16.2 & 0.78 & 18.00 & 1.14 \\
RXJ 0525.7+1205 & $15.6 \pm 6.3$ & 846.4 & $1.00 \pm 0.15$ & $0.62 \pm 0.13$ & 9.9 & 1.02 & 22.00 & 24.91 \\
RXJ 0528.4+1213 & $33.8 \pm 7.9$ & 661.9 & $1.00 \pm 0.05$ & $0.06 \pm 0.23$ & 43.3 & 0.61 & 21.70 & 28.14 \\
RXJ 0528.5+1219 & $11.0 \pm 4.4$ & 626.0 & $0.66 \pm 0.29$ & $0.30 \pm 0.37$ & 17.0 & 1.19 & 20.10 & 2.32 \\
RXJ 0528.9+1046 & $13.2 \pm 5.0$ & 639.2 & $1.00 \pm 0.16$ & $0.64 \pm 0.25$ & 21.4 & 1.05 & 22.00 & 26.86 \\
RXJ 0529.3+1210 & $23.2 \pm 6.9$ & 631.9 & $0.15 \pm 0.29$ & $0.03 \pm 0.42$ & 24.2 & 1.20 & 18.00 & 3.40 \\
RXJ 0530.9+1227 & $28.2 \pm 7.5$ & 693.4 & $1.00 \pm 0.06$ & $0.34 \pm 0.25$ & 23.2 & 0.51 & 22.00 & 94.99 \\
RXJ 0531.8+1218 & $10.5 \pm 4.8$ & 688.5 & $0.61 \pm 0.30$ & $-0.02 \pm 0.46$ & 7.9 & 0.84 & 19.40 & 1.42 \\
\hline
\end{tabular}

X-ray counts (background subtracted and vignetting corrected) detected in the broad ROSAT band, exposure time, hardness ratios, the maximum likelihood for existence of the source, and spectral fit results. Spectral fits are performed - as described in Neuhäuser et al. (1995b) using the hardness ratios and assuming a one-temperature Raymond-Smith model (Raymond \& Smith 1977). Both the emission energy $k T_{\mathrm{X}}$ and the absorbing foreground column density $N_{\mathrm{H}}$ are taken as free fit parameters. The procedure searches for the $\left(k T_{\mathrm{X}}, N_{\mathrm{H}}\right)$ pair that best fits the observed (HR1, HR2) pair. From the ROSAT PSPC response matrix and the fit results, we can then compute the individually spectral-corrected X-ray flux (also given in Table 1). The $\mathrm{X}$-ray source positions can be obtained from the positions of the optical counterparts and the offsets between X-ray and optical positions (given in Table 2).

In Table 2 we list the closest optical counterpart to each X-ray source which is sufficiently bright to be a potential PMS star at the distance of Taurus-Auriga (e.g., having $V$ brighter than $\sim 16 \mathrm{mag}$ ). All but two of the counterparts listed in Table 2 are GSC stars. This is a consequence of the selection process, as we have searched for GSC counterparts and used their $V$ magnitudes to select PMS candidates. The two non-GSC stars selected are listed in Simbad but, before our observations, it was not known whether they were TTS or not. In some cases, we have performed optical spectroscopy for several possible counterparts, when more than one star was found close to the X-ray position. As their $V$ magnitudes have not been used to estimate the likelihood that the relevant X-ray source may be a PMS star, we do not list these additional counterparts in Table 2, but in Table 3. If several optical counterparts are observed, we distinguish them by the letters at the end of the relevant ROSAT source designations (identifying the relative positions of the different counterparts). In Table 2 we list also the offset between the X-ray and optical positions (X - optical), the $V$ magnitude, the $\mathrm{X}$-ray to optical flux ratio, and the discrimination probability $P$, i.e. the value that reflects to which degree a source resembles typical TTS properties.

\section{Optical follow-up observations}

Medium-resolution spectra for the objects of our sample were obtained with the Intermediate Dispersion Spectrograph at the Cassegrain focus of the Isaac Newton Telescope in La Palma. The $500 \mathrm{~mm}$ camera, equipped with a TEK TK1024A CCD, was used in conjunction with the gratings $\mathrm{R} 600 \mathrm{R}$ and $\mathrm{R} 1200 \mathrm{Y}$, giving a resolution $\lambda / \Delta \lambda$ of about 4200 and 8400 , respectively. The observations were performed in October 1994, when both gratings were used, and in November 1995, when only the R1200 Y was used. Exposure times ranged from 200 to $1200 \mathrm{sec}-$ onds. Close to each stellar frame a wavelength calibration CuNe lamp was exposed; tungsten flat field exposure were taken both at the beginning and at the end of the night.

The CCD frames were reduced with the IRAF package $^{1}$. Each image was de-biased and flat fielded. The spectra were extracted with the IRAF task apall, which allows optimum extraction, cleaning from cosmic ray events, and background subtraction. Wavelength calibration was performed using the dispersion solution determined on the CuNe spectra by fitting an order two polynomial, with a rms of $0.03 \AA$ for the R $600 \mathrm{R}$ grating and $0.01 \AA$ for the R $1200 \mathrm{Y}$ grating.

Low-resolution spectra of additional objects were obtained at the European Southern Observatory (ESO) using the $1.52 \mathrm{~m}$ telescope equipped with a Boller \& Chivens spectrograph, in November 1995. A 900 grooves/mm (ESO

1 IRAF is distributed by the National Optical Observatory, which is operated by the Association of Universities for Research in Astronomy, Inc., under contract with the National Science Foundation. 
\# 5) grating and the CCD FORD $2048 L$ of $2048 \times 2048$ pixels were used. With this set-up a mean resolution of about $2.5 \AA$ (FWHM) in the $4600-7000 \AA$ spectral range was achieved. The reduction of these spectra was carried out using the MIDAS package. Bias and dark subtraction was first performed on each frame. The 2-D frames were then divided by a mean flat-field and then calibrated in wavelength. The sky subtraction, the extraction of one dimensional spectra and the flux calibration using a mean response function, were finally performed.

Spectral types have been assigned using the library of stellar spectra by Jacoby et al. (1984), available in digital form. For each of our stars a first guess of the spectral type was given by visual comparison. Then, we performed an iterative comparison in the following way: each spectrum was first normalized to the continuum; the spectral type standard spectrum was then normalized and rebinned to the same resolution and spectral range as the problem star; an overplot of the two spectra allowed us to compare directly different spectral features and reject or accept the guessed spectral type. Finally, we assigned the spectral type of the standard which follows most closely the features of the problem star. Particular attention was given to features like Ca I $6718 \AA$, Fe I blend $6495 \AA$ and $\mathrm{TiO}$ bands. The $\mathrm{H} \alpha$ line, in emission in most of our stars, was not used for the spectral classification. Except for the spectra obtained at ESO (which cover the 4700 to $6800 \AA$ spectral range), all of our spectra cover a short spectral range (from 6300 to $6800 \AA$ ). Bearing this in mind, we estimate that our classification can be uncertain to about one or two sub-classes and \pm 3 sub-classes for the stars earlier than about G5.

In Table 4, we present the results of our spectroscopic observations. We list all the counterparts observed (with names from Tables 2 and 3), identify the telescope, i.e. the resolution and wavelength range used, give equivalent widths of the $\mathrm{H} \alpha$ and $\mathrm{Li}$ I $\lambda 6708 \AA$ lines, and list the spectral types. In the lithium column, "no" means that no lithium line has been detected above the noise. Where possible, upper limits have been estimated (but not reported here), resulting always lower than $0.1 \AA$. The typical error in the Li equivalent width is $\pm 20 \mathrm{~m} \AA$, mainly due to uncertainties in the location of the continuum.

\section{Results}

Our aim is to search for low-mass PMS stars among the RASS source counterparts observed in this work, by studying their Li I $\lambda 6708 \AA$ doublet. The presence of a strong LiI $\lambda 6708 \AA$ absorption is the most evident indicator of the PMS nature. Typical equivalent widths range from $0.7 \AA$, observed in cTTS (Magazzù et al. 1992) to $0.1 \AA$, observed in wTTS (Martín et al. 1994), while equivalent widths lower than about $0.3 \AA$ have been measured in Pleiades objects (Soderblom et al. 1993; García López et al. 1994).

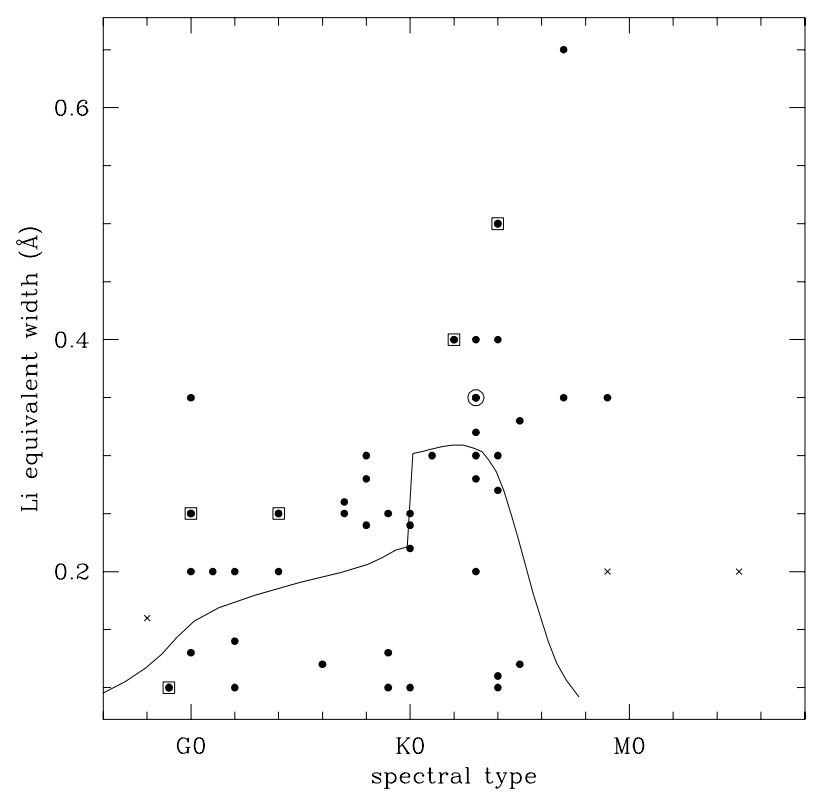

Fig. 3. Equivalent widths of the Li I $\lambda 6708 \AA$ doublet vs. spectral type for the stars in our sample. The continuous line has been adapted from Fig. 2d in Soderblom et al. (1993) and represents the upper envelope of $\mathrm{Li}$ equivalent width for Pleiades stars. Squares (circles) indicate two (three) points in the same position. Crosses are doubtful points (see text)

In Fig. 3 we show, for our objects in which $\mathrm{Li}$ has been detected, the $\operatorname{Li} I \lambda 6708 \AA$ equivalent width vs. the spectral type. We plot also the upper envelope of Li I $\lambda 6708 \AA$ equivalent widths for stars in the Pleiades (adapted from Fig. 2d in Soderblom et al. 1993).

In this paper we classify as low-mass PMS stars those objects, later than F7, satisfying the following criterion:

The Li I $\lambda 6708 \AA$ equivalent width must be greater than in Pleiades objects of the same spectral type.

In other words, a star is an PMS if it can be located above the continuous line in Fig. 3. Stars in which lithium has been detected, but below the Pleiades upper envelope, will be classified as possible PMS stars ("PMS?" in Table 4). Objects with no lithium will be classified as dKe or dMe stars, depending on their spectral type, if their $\mathrm{H} \alpha$ is in emission or strongly filled in. The remaining objects will be classified as "non-PMS". We are aware that this classification is somewhat arbitrary, for example among the non-PMS we could find some post-TTS, which in some cases show the Li resonance doublet weaker than $0.1 \AA$ (Martín et al. 1992). However, we believe this is a conservative classification, which makes us quite confident that objects classified as "PMS" are really PMS stars. In order to get accurate ages of our objects, we are planning further observations, aimed to locate our PMS candidates in the $\mathrm{H}-\mathrm{R}$ diagram and measure their lithium abundance. 
Table 2. Closest optical counterparts. Listed is the closest optical counterpart to each X-ray source in Table 1 . We list designation (as in Table 1, but with indications of the particular counterpart meant here, if several counterparts have been observed), optical position (for J 2000.0) from the GSC (or Simbad if available), offset between X-ray and optical position, $V$ magnitude from the GSC (or Simbad as indicated by colons if available), X-ray to optical flux ratio, discrimination probability $P$, and remarks. Note that some GSC counterparts appear on several GSC plates which may have different colors and different filters; we have always chosen the closest counterpart, i.e. we neither try to average the magnitudes nor indicate the plate color as GSC magnitudes are very uncertain anyway (about half a magnitude)

\begin{tabular}{|c|c|c|c|c|c|c|c|c|}
\hline designation & $\alpha$ (opt.) & $\delta$ (opt.) & $\Delta \alpha\left({ }^{\prime \prime}\right)$ & $\Delta \delta\left(^{\prime \prime}\right)$ & $V$ & $\log \left(f_{\mathrm{X}} / f_{\mathrm{V}}\right)$ & $P$ & remarks \\
\hline RXJ 0207.0-1407 & 20702.94 & -140652.0 & -33.0 & -5.8 & 15.1 & -0.83 & 0.65 & \\
\hline RXJ 0209.1-1536 & 20906.70 & -153543.0 & -3.6 & 0.7 & 13.0 & -1.32 & 0.81 & \\
\hline RXJ 0210.4-1308NE & 21025.84 & -130757.0 & 3.0 & 4.9 & 10.5 & -2.29 & 0.76 & \\
\hline RXJ 0212.3-1330 & 21218.72 & -133042.1 & 17.6 & -17.3 & 11.4 & -2.01 & 0.65 & \\
\hline RXJ 0215.0-1402 & 21500.45 & -140131.9 & 13.8 & 31.9 & 15.1 & -0.77 & 0.54 & \\
\hline RXJ 0218.6-1004 & 21839.54 & -100405.0 & -12.3 & 6.3 & 11.8 & -1.79 & 0.81 & \\
\hline RXJ 0219.4-1321B & 21925.30 & -132106.6 & 9.6 & -12.0 & 14.8 & -0.81 & 0.65 & \\
\hline RXJ 0219.7-1026 & 21947.38 & -102539.9 & -9.3 & 6.9 & 11.6 & -1.50 & 0.70 & \\
\hline RXJ 0220.4-1305 & 22028.34 & -130523.9 & 0.5 & -18.8 & 14.6 & -0.75 & 0.27 & \\
\hline RXJ $0223.3-1615 \mathrm{NE}$ & 22321.41 & -161420.9 & -5.3 & 12.8 & 13.4 & -1.51 & 0.86 & \\
\hline RXJ 0229.5-1224 & 22935.05 & -122408.8 & 13.0 & -13.6 & 9.9: & -2.74 & 0.54 & HD 15526 \\
\hline RXJ 0237.3-0527 & 23719.85 & -052654.1 & 4.6 & 7.6 & 13.3 & -1.24 & 0.54 & \\
\hline RXJ 0239.1-1028 & 23908.72 & -102744.1 & -0.6 & 2.7 & 13.2 & -1.50 & 0.65 & \\
\hline RXJ 0243.9-0850 & 24352.29 & -084930.3 & 18.3 & 14.2 & 12.9 & -1.53 & 0.59 & \\
\hline RXJ 0248.3-1117 & 24822.16 & -111712.3 & 25.8 & 6.4 & 12.0 & -2.00 & 0.65 & \\
\hline RXJ 0251.8-0203 & 25148.47 & -020338.1 & 11.1 & -8.9 & 12.7 & -1.58 & 0.76 & \\
\hline RXJ 0254.8-0709SE & 25452.43 & -070924.2 & 4.7 & 5.8 & 14.5 & -0.78 & 0.59 & \\
\hline RXJ 0255.8-0750S & 25552.63 & -075039.0 & -3.4 & -15.3 & 14.0 & -1.06 & 0.81 & \\
\hline RXJ $0309.1+0324 \mathrm{~N}$ & 30909.86 & 032344.4 & 25.7 & 5.6 & 10.2 & -2.33 & 0.59 & \\
\hline RXJ $0312.8-0414 \mathrm{NW}$ & 31250.43 & -041408.1 & 20.8 & -18.4 & 9.9 & -2.22 & 0.76 & \\
\hline RXJ 0314.8-0406 & 31450.70 & -040556.4 & -14.6 & -5.5 & 14.4 & -1.16 & 0.70 & \\
\hline RXJ 0317.9+0231 & 31759.18 & 023012.9 & 11.6 & 32.9 & 11.0 & -2.26 & 0.70 & \\
\hline RXJ 0319.3+0003 & 31922.53 & 000232.4 & -0.2 & 17.6 & 9.2: & -3.24 & 0.27 & SAO 130417, (1) \\
\hline RXJ 0324.4+0231 & 32425.22 & 023101.2 & 3.6 & -7.7 & 12.4 & -1.45 & 0.65 & \\
\hline RXJ 0329.1+0118 & 32908.01 & 011805.3 & -15.0 & 11.4 & 10.7 & -2.52 & 0.65 & \\
\hline RXJ $0330.7+0306 \mathrm{~N}$ & 33043.45 & 030547.9 & 12.4 & 2.6 & 10.9 & -1.86 & 0.81 & \\
\hline RXJ 0333.0+0354 & 33301.54 & 035338.4 & 3.0 & 14.9 & 13.3 & -1.55 & 0.70 & \\
\hline RXJ 0333.1+1036 & 33311.62 & 103556.3 & -9.6 & 9.8 & 11.7 & -1.84 & 0.65 & \\
\hline RXJ 0336.0+0846 & 33600.32 & 084535.7 & 15.4 & 0.6 & 12.4 & -1.94 & 0.76 & \\
\hline RXJ 0338.1+1224 & 33809.04 & 122353.1 & 16.6 & 0.6 & 10.9 & -2.55 & 0.65 & \\
\hline RXJ 0338.3+1020 & 33818.21 & 102017.1 & 21.6 & -6.2 & 11.0 & -2.30 & 0.70 & \\
\hline RXJ 0339.6+0624 & 33940.57 & 062443.1 & 13.5 & -17.3 & 11.3 & -2.38 & 0.70 & \\
\hline RXJ $0340.3+1220$ & 34019.26 & 122018.0 & -1.4 & -0.3 & 12.5 & -1.36 & 0.76 & \\
\hline RXJ 0340.5+0639 & 34031.48 & 063900.3 & -15.4 & 11.8 & 13.2 & -1.79 & 0.81 & \\
\hline RXJ $0341.2+0453$ & 34114.30 & 045325.4 & 3.2 & -11.0 & 15.2 & -0.17 & 0.70 & \\
\hline RXJ 0341.2+0759 & 34114.36 & 075933.1 & 7.1 & -3.7 & 10.7 & -2.65 & 0.70 & \\
\hline RXJ 0343.6+1039 & 34340.51 & 103914.0 & -1.9 & -9.3 & 10.2 & -2.50 & 0.65 & \\
\hline RXJ 0344.8+0359 & 34453.15 & 035930.9 & 9.0 & 5.6 & 12.3 & -1.50 & 0.81 & \\
\hline RXJ 0347.2+0933SW & 34714.25 & 093253.0 & 21.5 & -1.4 & 13.4 & -1.24 & 0.65 & \\
\hline RXJ 0347.9+0616 & 34756.81 & 061607.0 & 19.1 & -4.8 & 11.0 & -2.40 & 0.70 & \\
\hline RXJ 0348.2+1109 & 34816.50 & 110841.0 & -4.6 & -0.8 & 10.1: & -2.34 & 0.70 & HD $23793 \mathrm{~B},(2)$ \\
\hline RXJ 0348.5+0832 & 34831.42 & 083137.5 & 15.9 & -7.5 & 10.9 & -2.04 & 0.76 & \\
\hline RXJ $0349.4+1255 \mathrm{~N}$ & 34927.85 & 125441.4 & -6.5 & 6.1 & 9.1: & -2.83 & 0.65 & $\mathrm{BD}+12511$ \\
\hline RXJ 0350.2+0849 & 35012.91 & 084934.0 & -4.5 & -6.1 & 12.1 & -2.09 & 0.81 & \\
\hline RXJ 0350.6+0454 & 35041.72 & 045344.7 & -28.8 & -3.4 & 15.2 & -0.82 & 0.76 & \\
\hline RXJ 0350.6+1033 & 35040.80 & 103251.1 & -9.3 & 19.9 & 10.1 & -2.70 & 0.59 & \\
\hline RXJ 0351.4+0953W & 35126.30 & 095337.0 & 2.6 & -16.5 & 12.6 & -1.23 & 0.65 & \\
\hline RXJ $0351.8+0413$ & 35149.40 & 041330.7 & -16.5 & -12.3 & 13.5 & -1.51 & 0.81 & \\
\hline RXJ 0352.4+1223 & 35224.68 & 122244.2 & -4.2 & -6.9 & 9.5: & -2.93 & 0.59 & $\mathrm{BD}+11533$ \\
\hline
\end{tabular}


Table 2. continued

\begin{tabular}{|c|c|c|c|c|c|c|c|c|}
\hline designation & $\alpha$ (opt.) & $\delta$ (opt.) & $\Delta \alpha\left({ }^{\prime \prime}\right)$ & $\Delta \delta\left(^{\prime \prime}\right)$ & $V$ & $\log \left(f_{\mathrm{X}} / f_{\mathrm{V}}\right)$ & $P$ & remarks \\
\hline RXJ 0354.1+0528 & 35406.58 & 052723.7 & 11.3 & 20.8 & 11.9 & -2.18 & 0.70 & \\
\hline RXJ 0354.3+0535 & 35421.28 & 053540.9 & -0.5 & -14.2 & 10.1 & -2.51 & 0.54 & \\
\hline RXJ 0354.4+1204 & 35425.15 & 120407.9 & -3.3 & -14.9 & 11.2 & -2.36 & 0.70 & \\
\hline RXJ $0354.8+1232$ & 35450.70 & 123206.4 & 4.9 & 10.7 & 12.9 & -1.40 & 0.81 & \\
\hline RXJ 0356.7+0943 & 35645.51 & 094339.4 & -4.0 & -13.2 & 13.3 & -1.44 & 0.70 & \\
\hline RXJ $0357.3+1258$ & 35721.34 & 125817.3 & 3.8 & 3.6 & 10.9 & -2.16 & 0.70 & \\
\hline RXJ 0358.1+0932 & 35812.62 & 093221.6 & -13.5 & -2.1 & 12.2 & -1.42 & 0.70 & \\
\hline RXJ 0400.0+0730 & 40001.14 & 073010.4 & -0.7 & 4.0 & 12.2 & -1.86 & 0.65 & \\
\hline RXJ $0400.1+0818 \mathrm{~N}$ & 40009.37 & 081819.3 & 6.6 & 4.0 & 9.9: & -1.54 & 0.65 & $\mathrm{BD}+07582 \mathrm{~B},(3)$ \\
\hline RXJ $0400.8+1116$ & 40053.92 & 111527.8 & -25.2 & 21.9 & 13.8 & -1.07 & 0.70 & \\
\hline RXJ 0402.5+0552 & 40235.68 & 055136.0 & -18.0 & 14.5 & 10.9 & -2.08 & 0.70 & \\
\hline RXJ $0403.5+0837$ & 40329.69 & 083714.2 & 35.6 & 4.7 & 12.6 & -1.42 & 0.76 & \\
\hline RXJ 0404.4+0519 & 40428.48 & 051843.7 & -5.1 & 4.0 & 10.9 & -2.02 & 0.81 & \\
\hline RXJ 0405.5+0324 & 40530.24 & 032350.4 & -2.1 & 6.5 & 11.5 & -1.61 & 0.81 & \\
\hline RXJ $0407.2+0113 \mathrm{~N}$ & 40716.49 & 011314.5 & 15.2 & -4.3 & 10.5 & -2.50 & 0.54 & \\
\hline RXJ 0407.6+0638 & 40737.26 & 063845.2 & 9.5 & -23.1 & 14.8 & -0.56 & 0.54 & \\
\hline RXJ 0408.6+1017 & 40839.90 & 101732.6 & -13.5 & -26.8 & 12.6 & -1.84 & 0.65 & \\
\hline RXJ 0408.8+1028 & 40849.57 & 102750.0 & -3.8 & -4.3 & 8.9: & -2.08 & 0.70 & HD 26172 \\
\hline RXJ 0409.8+1209 & 40951.54 & 120902.3 & 22.0 & -7.6 & 12.0 & -1.81 & 0.65 & HD 286556 \\
\hline RXJ 0410.6+0608 & 41039.66 & 060838.9 & -22.2 & -19.8 & 12.9 & -1.56 & 0.86 & \\
\hline RXJ $0413.2+1028$ & 41314.66 & 102801.1 & -10.7 & -14.7 & 14.9 & -0.56 & 0.65 & \\
\hline RXJ 0418.6+0143 & 41839.27 & 014210.5 & 13.5 & 29.9 & 12.3 & -1.83 & 0.59 & \\
\hline RXJ 0419.8+0214 & 41949.92 & 021343.5 & -13.8 & -11.3 & 14.6 & -0.61 & 0.76 & \\
\hline RXJ 0419.9+0231 & 41959.60 & 023030.6 & -11.5 & 24.1 & 13.6 & -0.54 & 0.76 & \\
\hline RXJ 0422.9+0141 & 42254.61 & 014132.1 & 22.5 & -17.7 & 12.3 & -1.63 & 0.70 & \\
\hline RXJ 0423.5+0955 & 42330.28 & 095430.0 & 10.6 & 13.8 & 11.6 & -1.66 & 0.49 & \\
\hline RXJ 0425.5+1210 & 42535.30 & 121000.0 & -6.3 & -0.5 & 10.4 & -2.40 & 0.65 & HD 286753 \\
\hline RXJ 0426.4+0957W & 42626.77 & 095659.7 & 11.6 & -3.4 & 12.0 & -1.91 & 0.81 & \\
\hline RXJ 0427.4+1039 & 42730.29 & 103848.9 & -11.1 & 1.7 & 11.3 & -2.05 & 0.65 & \\
\hline RXJ $0427.5+0616$ & 42732.07 & 061552.1 & 0.4 & 1.8 & 10.6 & -1.80 & 0.76 & \\
\hline RXJ $0427.8+0049$ & 42752.84 & 004925.9 & -5.5 & 3.3 & 9.0: & -2.92 & 0.22 & $\mathrm{BD}+00760,(4)$ \\
\hline RXJ 0429.9+0155 & 42956.94 & 015448.9 & 0.8 & 12.0 & 11.1 & -2.19 & 0.70 & \\
\hline RXJ $0433.7+0522$ & 43346.09 & 052209.1 & 18.8 & -16.6 & 15.3 & -0.71 & 0.81 & \\
\hline RXJ $0434.3+0226$ & 43419.51 & 022626.1 & 5.8 & 2.1 & 13.3 & -1.12 & 0.86 & \\
\hline RXJ 0435.5+0455 & 43531.56 & 045532.3 & -8.9 & -12.6 & 9.7 & -2.78 & 0.49 & \\
\hline RXJ 0441.9+0537 & 44157.64 & 053634.3 & 0.3 & 7.3 & 10.2: & -1.78 & 0.70 & $\mathrm{BD}+05706$ \\
\hline RXJ 0442.3+0118 & 44218.61 & 011739.9 & 8.9 & 3.5 & 11.2 & -2.03 & 0.70 & \\
\hline RXJ 0442.5+0906 & 44231.94 & 090601.7 & -16.5 & 10.1 & 10.6: & -1.94 & 0.76 & $\mathrm{BD}+08742$ \\
\hline RXJ 0442.6+1018 & 44240.81 & 101744.8 & -3.6 & 3.3 & 8.2 & -2.59 & 0.65 & \\
\hline RXJ 0442.9+0400 & 44254.69 & 040011.7 & 9.6 & 1.7 & 10.9 & -2.21 & 0.70 & \\
\hline RXJ 0444.3+0941 & 44420.21 & 094106.8 & 1.5 & 0.3 & 8.6: & -2.60 & 0.65 & HD 287017 \\
\hline RXJ 0444.4+0725 & 44427.16 & 072459.8 & -9.3 & 8.7 & 13.7 & -1.22 & 0.65 & \\
\hline RXJ $0444.7+0814$ & 44445.44 & 081347.6 & -20.1 & 4.5 & 11.8 & -1.86 & 0.70 & \\
\hline RXJ 0445.2+0729 & 44513.18 & 072917.7 & -9.9 & 10.4 & 12.2 & -1.97 & 0.81 & \\
\hline RXJ 0445.3+0914 & 44523.81 & 091347.8 & -4.9 & -13.0 & 11.8 & -2.15 & 0.65 & \\
\hline RXJ 0445.5+1207 & 44536.49 & 120751.1 & -7.4 & -22.4 & 12.9 & -1.74 & 0.76 & \\
\hline RXJ 0448.0+0738 & 44800.86 & 073756.9 & -5.6 & -7.0 & 11.2 & -1.40 & 0.70 & \\
\hline RXJ 0450.0+0151 & 45004.68 & 015043.1 & 17.0 & 13.2 & 12.2 & -1.63 & 0.86 & \\
\hline RXJ 0451.6+0619 & 45141.51 & 061920.6 & -12.4 & -18.1 & 12.8 & -1.73 & 0.86 & \\
\hline RXJ 0459.9+1017 & 45954.95 & 101718.2 & -12.6 & 0.9 & 14.4 & -0.62 & 0.76 & \\
\hline RXJ 0511.2+1031 & 51115.98 & 103036.0 & -1.2 & 8.9 & 14.0 & -1.02 & 0.86 & \\
\hline RXJ $0511.9+1112$ & 51200.30 & 111219.8 & -9.5 & -14.1 & 11.4 & -2.21 & 0.65 & \\
\hline RXJ 0512.0+1020 & 51203.21 & 102006.8 & -7.5 & 5.3 & 11.3 & -1.99 & 0.76 & \\
\hline RXJ 0513.6+0955 & 51340.44 & 095450.7 & -13.5 & 2.3 & 12.0 & -2.08 & 0.65 & \\
\hline
\end{tabular}


Table 2. continued

\begin{tabular}{|c|c|c|c|c|c|c|c|c|}
\hline designation & $\alpha$ (opt.) & $\delta$ (opt.) & $\Delta \alpha\left({ }^{\prime \prime}\right)$ & $\Delta \delta\left(^{\prime \prime}\right)$ & $V$ & $\log \left(f_{\mathrm{X}} / f_{\mathrm{V}}\right)$ & $P$ & remarks \\
\hline RXJ 0515.3+1221 & 51520.55 & 122114.1 & 12.6 & -1.1 & 11.6 & -1.84 & 0.81 & \\
\hline RXJ 0516.3+1148 & 51621.52 & 114747.3 & 5.3 & -3.2 & 12.4 & -1.53 & 0.86 & \\
\hline RXJ 0523.0+0934 & 52304.65 & 093401.0 & -25.2 & 5.0 & 10.4 & -2.64 & 0.76 & \\
\hline RXJ 0523.5+1005 & 52333.72 & 100429.8 & -1.5 & -11.1 & 11.2 & -1.81 & 0.76 & \\
\hline RXJ 0523.9+1101 & 52357.04 & 110057.5 & -26.4 & 15.9 & 10.8 & -2.62 & 0.70 & \\
\hline RXJ 0525.7+1205SE & 52547.21 & 120410.2 & 0.6 & 36.3 & 14.1 & -1.17 & 0.65 & \\
\hline RXJ 0528.4+1213 & 52825.74 & 121236.1 & -4.9 & 5.9 & 11.5 & -1.77 & 0.81 & \\
\hline RXJ 0528.5+1219 & 52835.19 & 121904.0 & 1.8 & 10.1 & 12.7 & -1.77 & 0.70 & \\
\hline RXJ 0528.9+1046 & 52858.50 & 104537.9 & -7.9 & -0.1 & 12.7 & -1.71 & 0.76 & \\
\hline RXJ 0529.3+1210 & 52918.97 & 120929.7 & -4.6 & 3.6 & 12.9 & -1.36 & 0.54 & \\
\hline RXJ $0530.9+1227$ & 53057.23 & 122726.8 & 27.3 & 0.4 & 10.7 & -2.19 & 0.70 & \\
\hline RXJ $0531.8+1218$ & 53147.77 & 121808.1 & 16.4 & 12.8 & 12.1 & -2.06 & 0.81 & \\
\hline
\end{tabular}

Remarks: (1) Not listed in the GSC; (2) HD 23793 B (F5V) and the early-type star HR 1174 (B3) are an optical pair with 9" separation (Lindroos 1986); the later type star is more likely to be the true optical counterpart of the X-ray source (e.g. Schmitt et al. 1993); (3) Companion $\mathrm{N}$ is an unresolved binary itself; (4) Not listed in the GSC.

Table 3. Other optical counterparts observed. Listed are optical counterparts other than those in Table 2, but which have also been observed by us. We list designation (as in Table 1, but with indications regarding the particular counterpart listed here), approximate optical position (for J 2000.0), a remark regarding its position relative to the counterpart listed in Table 2, approximate $V$ magnitude, and remarks. The source for optical positions, $V$ magnitudes, and remarks are either GSC, Simbad, or visual inspection of plates

\begin{tabular}{lrrlll}
\hline designation & $\alpha$ (opt.) & $\delta$ (opt.) & rel. pos. & $V$ & remarks \\
\hline RXJ 0210.4-1308SW & 21026 & -130756 & $2^{\prime \prime}$ southwest of NE & 12.5 & \\
RXJ 0219.4-1321A & 22029 & -132025 & $60^{\prime \prime}$ northeast of B & 13 & \\
RXJ 0219.4-1321C & 21925 & -132147 & $40^{\prime \prime}$ south of B & 15.5 & \\
RXJ 0223.3-1615SW & 22325 & -161431 & $10^{\prime \prime}$ southwest of NE & 13.5 & \\
RXJ 0254.8-0709NW & 25453 & -070920 & $6^{\prime \prime}$ northwest of SE & 15.5 & \\
RXJ 0255.8-0750N & 25552 & -075029 & \multicolumn{1}{l}{$10^{\prime \prime}$ north of S } & 15.5 & \\
RXJ 0309.1+0324S & 30910 & 032342 & $2^{\prime \prime}$ south of N & 11 & \\
RXJ 0312.8-0414SE & 31251 & -041419 & $14^{\prime \prime}$ southeast of NW & 10.5 & \\
RXJ 0330.7+0306S & 33043 & 030518 & $30^{\prime \prime}$ south of N & 15 & \\
RXJ 0347.2+0933NE & 34717 & 093308 & $40^{\prime \prime}$ northeast of SW & 12 & \\
RXJ 0349.4+1255S & 34928 & 125428 & $14^{\prime \prime}$ south of N & 10 & BD+12 511B \\
RXJ 0351.4+0953E & 35128 & 095334 & $25^{\prime \prime}$ east of W & 13.7 & \\
RXJ 0400.1+0818S & 40009 & 081815 & $4^{\prime \prime}$ south of N & 10 & BD+07 582 \\
RXJ 0407.2+0113S & 40716 & 011312 & $2^{\prime \prime}$ south of N & 12 & \\
RXJ 0426.4+0957E & 42730 & 095700 & $45^{\prime \prime}$ east of W & 11.5 & also in GSC \\
RXJ 0525.7+1205NW & 52544 & 120430 & $45^{\prime \prime}$ northwest of SE & 14.5 & \\
\hline
\end{tabular}

Note that we do not impose any restriction on $\mathrm{H} \alpha$ for PMS stars. In fact, TTS show a wide range of $\mathrm{H} \alpha$ equivalent widths, from strong emission in cTTS to practically no emission in post-TTS. However, from Table 4 we can see that most of the objects classified as PMS or PMS? show weak $\mathrm{H} \alpha$ emission or $\mathrm{H} \alpha$ absorption shallower than in main sequence objects, as expected for wTTS.

Although the objects RXJ 0255.8-0750N, RXJ 0333.0 +0354 , and RXJ $0422.9+0141$ in Fig. 3 are located above the Pleiades upper envelope, in this paper we classify them as PMS? For the first two objects this is due to the quite high level of noise, which makes doubtful the detection of the lithium line. For RXJ 0422.9+0141 we note that this object is a spectroscopic binary (see remark 7 in Table 4) and the determination of the spectral type of its components is rather uncertain.

In total, there are 115 RASS sources selected for optical follow-up observations. As some RASS sources have several potential optical counterparts, we have performed spectroscopy for a total of 131 stars, all listed in Table 4. 
$0255.8-0750$
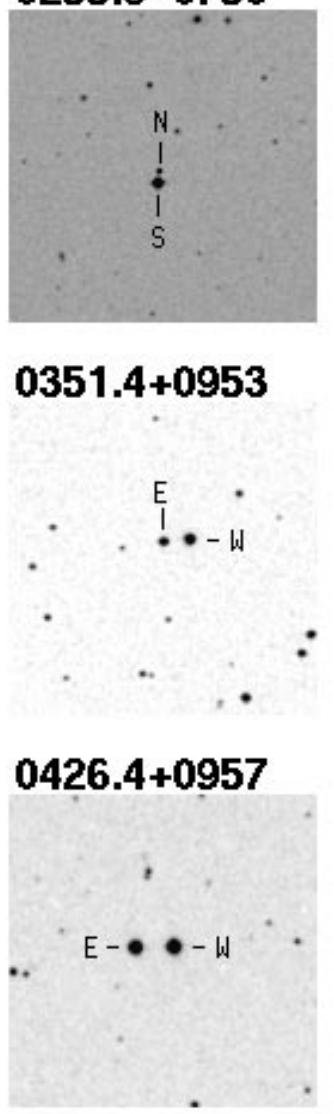
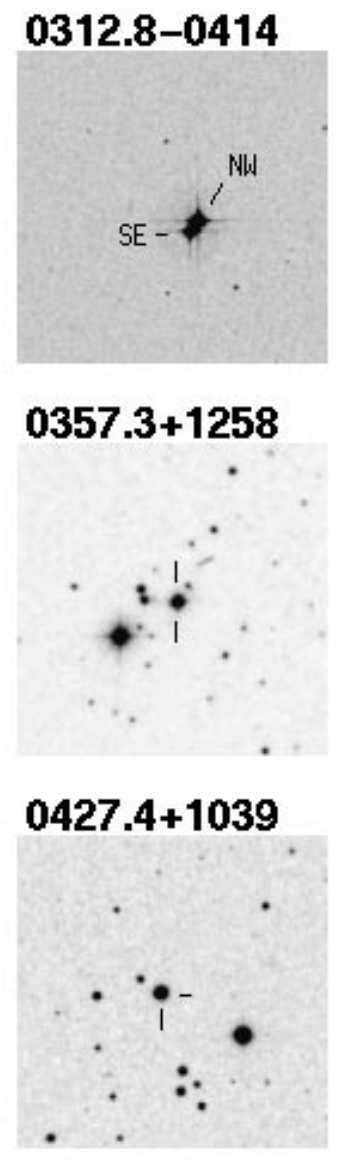

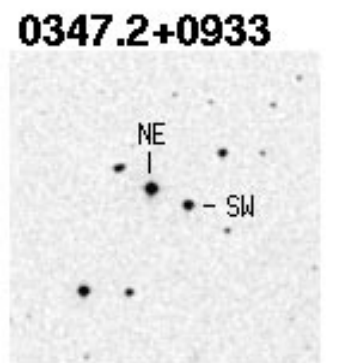

$0347.9+0616$

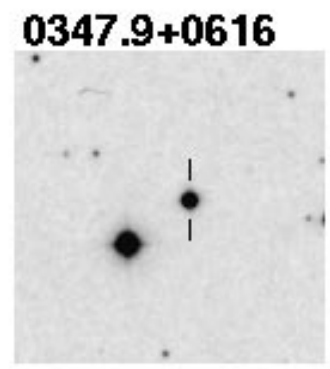

$0400.1+0818$.

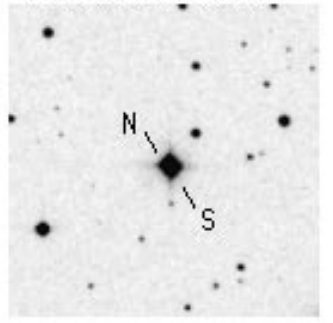

$0445.5+1207$

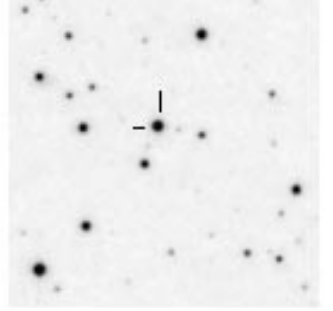

$0407.2+0113$

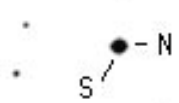

\section{$0516.3+1148$}

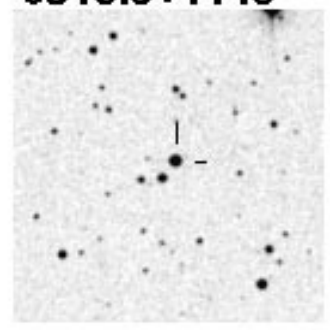

Fig. 4. Some finding charts $\left(5^{\prime} \times 5^{\prime}\right)$. North is up, East on the left. The other PMS and PMS? stars can be easily identified (see text)

In Fig. 4 we show the finding charts for some object whose identification may be difficult; all the other new PMS can be easily identified, as they are GSC stars with no bright stars in the vicinity. In case of need, the charts for any object observed here are available on request.

There are 30 stars in our sample which can be classified as PMS stars, according to our criterion. These objects are counterparts to 28 RASS sources, i.e. for two RASS sources we have found two new PMS stars each (with separations of $2^{\prime \prime}$ and $\left.14^{\prime \prime}\right)$. In addition, there are 19 RASS counterparts classified as PMS? as well as 17 and 5 RASS counterparts classified as $\mathrm{dKe}$ and $\mathrm{dMe}$, respectively. In several spectra, we clearly see double lines indicative of close binaries (identified as spectroscopic binaries - "SB" - in Table 4). Namely, we find seven (one) SB among the stars classified as dKe (dMe) stars, three among the PMS? stars, and four among the non-PMS stars.

The spectra of all 30 stars classified in Table 4 as new PMS stars are shown in Fig. 5. These and all the other spectra are available from the authors. In some cases one can see quite broad lines indicative of either close binaries and/or fast rotation. As some relatively fast rotators are expected to be present among TTS, we do not classify such PMS stars as SB.
As can be seen from Table 4, three of the 15 stars classified as wTTS in Neuhäuser et al. (1995c) may not be real young PMS stars according to our stricter criterion; here they are classified as dKe stars. Seven other stars classified as wTTS by Neuhäuser et al. are now classified as PMS? stars, while the other five wTTS are confirmed to be PMS stars also in this paper.

We note that one of the stars studied here (HD 23793 B) is the late-type secondary in the so-called Lindroos sample (Lindroos 1986), a set of double stars with the primary being an early-type star and the secondary being late-type. It has been suggested that many of the secondaries in this sample may be post-TTS (Lindroos 1986). However, both Pallavicini et al. (1992) and Martín et al. (1992) consistently found that only about one third of them may be genuine post-TTS. As far as HD 23793 $\mathrm{B}$ is concerned (spectral type F5), both Pallavicini et al. (1992) and ourselves find $\mathrm{H} \alpha$ absorption and very weak Li I $\lambda 6708 \AA$. Pallavicini et al. (1992) did not detect $\mathrm{Ca} \mathrm{H}$ and $\mathrm{K}$ emission and classified this Lindroos pair as an optical system. According to our criterion, we classify this star as non-PMS as well. 
Table 4. Results of medium-resolution spectroscopy of all potential counterparts to RASS X-ray sources. Listed are source designation, the telescope used to observe the star (INT for the Isaac Newton Telescope, ESO for the ESO $1.52 \mathrm{~m}$ telescope), H $\alpha$ equivalent width (negative when in emission, "f" indicates that $\mathrm{H} \alpha$ emission is filling in the absorption line, "abs" for absorption without available equivalent width), lithium $6708 \AA$ absorption equivalent width (colons indicate large uncertainty), spectral type (always luminosity class V unless otherwise noted), nature of object (see text), and remarks (e.g. "SB" for spectroscopic binaries or "(N95c)" if mentioned in Neuhäuser et al. 1995c)

\begin{tabular}{|c|c|c|c|c|c|c|}
\hline designation & tel. & $\begin{array}{r}W_{\lambda}(\mathrm{H} \alpha) \\
(\AA)\end{array}$ & $\begin{array}{r}W_{\lambda}(\mathrm{Li}) \\
(\AA)\end{array}$ & SpType & nature & remarks \\
\hline RXJ 0207.0-1407 & INT & 3.3 & no & F8 & non-PMS & \\
\hline RXJ 0209.1-1536 & INT & -1.2 & no & K4 & $\mathrm{dKe}$ & \\
\hline RXJ 0210.4-1308SW & INT & -0.12 & no & K3 & $\mathrm{dKe}$ & $2^{\prime \prime}$ sep. \\
\hline RXJ $0210.4-1308 \mathrm{NE}$ & INT & 3.5 & no & G5 & non-PMS & $2^{\prime \prime}$ sep. \\
\hline RXJ 0212.3-1330 & INT & 1.2 & no & K1 & non-PMS & \\
\hline RXJ 0215.0-1402 & INT & 1.15 & no & K4 & non-PMS & maybe K3 III \\
\hline RXJ 0218.6-1004 & INT & 1.6 & no & G8 & non-PMS & $\mathrm{SB}$ \\
\hline RXJ $0219.4-1321 \mathrm{C}$ & $\mathrm{ESO}$ & abs & no & G0 & non-PMS & \\
\hline RXJ 0219.4-1321B & $\mathrm{ESO}$ & $0.60 \mathrm{f}$ & no & M0 & $\mathrm{dMe}$ & \\
\hline RXJ 0219.4-1321A & ESO & -4.30 & no & M3 & $\mathrm{dMe}$ & \\
\hline RXJ 0219.7-1026 & INT & -0.25 & 0.1 : & K4 & PMS? & $(\mathrm{N} 95 \mathrm{c}),(1)$ \\
\hline RXJ 0220.4-1305 & INT & 5.7 & no & F0? & non-PMS & \\
\hline RXJ 0223.3-1615SW & $\mathrm{ESO}$ & -0.8 & no & $\mathrm{K} 7$ & $\mathrm{dKe}$ & $10^{\prime \prime}$ sep. \\
\hline RXJ $0223.3-1615 \mathrm{NE}$ & $\mathrm{ESO}$ & 2.1 & no & G7 & non-PMS & $10^{\prime \prime}$ sep. \\
\hline RXJ 0229.5-1224 & $\mathrm{ESO}$ & 2.6 & 0.28 & G8 & PMS & HD 15526 \\
\hline RXJ 0237.3-0527 & INT & -0.30 & no & K5 & $\mathrm{dKe}$ & $(\mathrm{N} 95 \mathrm{c})$ \\
\hline RXJ 0239.1-1028 & INT & -0.35 & no & K7-M0 & $\mathrm{dKe}$ & $\mathrm{SB}$ \\
\hline RXJ 0243.9-0850 & $\mathrm{ESO}$ & $0.50 \mathrm{f}$ & no & M2 & $\mathrm{dMe}$ & \\
\hline RXJ 0248.3-1117 & INT & 2.1 & no & G7 & non-PMS & \\
\hline RXJ 0251.8-0203 & INT & -0.60 & no & K6 & $\mathrm{dKe}$ & (N95c) \\
\hline RXJ 0254.8-0709NW & INT & -4.2 & no & M5 & $\mathrm{dMe}$ & $3^{\prime \prime}$ sep. \\
\hline RXJ 0254.8-0709SE & INT & -2.2 & no & M3 & $\mathrm{dMe}$ & $3^{\prime \prime}$ sep. \\
\hline RXJ 0255.8-0750N & INT & -0.7 & 0.2 : & M5 & PMS? & $10^{\prime \prime}$ sep. \\
\hline RXJ 0255.8-0750S & INT & -0.2 & no & K7-M0 & $\mathrm{dKe}$ & $10^{\prime \prime}$ sep., SB \\
\hline RXJ $0309.1+0324 \mathrm{~N}$ & INT & 3.6 & no & F7 & non-PMS & $2^{\prime \prime}$ sep. \\
\hline RXJ 0309.1+0324S & INT & 1.7 & no & G6 & non-PMS & $2^{\prime \prime}$ sep. \\
\hline RXJ 0312.8-0414NW & $\mathrm{ESO}$ & 3.5 & 0.2 & G0 & PMS & $14^{\prime \prime}$ sep. \\
\hline RXJ 0312.8-0414SE & $\mathrm{ESO}$ & 2.5 & 0.3 & G8 & PMS & $14^{\prime \prime}$ sep. \\
\hline RXJ 0314.8-0406 & INT & 5.5 & no & late $\mathrm{A}$ & non-PMS & \\
\hline RXJ 0317.9+0231 & INT & 1.4 & no & G6 & non-PMS & \\
\hline RXJ 0319.3+0003 & $\mathrm{ESO}$ & 4.4 & no & G5 & non-PMS & SAO 130417 \\
\hline RXJ 0324.4+0231 & INT & -0.40 & 0.33 & K5 & PMS & $(\mathrm{N} 95 \mathrm{c})$ \\
\hline RXJ 0329.1+0118 & INT & 4.0 & 0.13 & G0 & PMS? & $(\mathrm{N} 95 \mathrm{c})$ \\
\hline RXJ $0330.7+0306 \mathrm{~N}$ & INT & $1.1 \mathrm{f}$ & no & K5 & $\mathrm{dKe}$ & $\mathrm{SB},(2)$ \\
\hline RXJ $0330.7+0306 \mathrm{~S}$ & INT & 1.0 & no & K7 & non-PMS & \\
\hline RXJ 0333.0+0354 & INT & -2.4 & $0.2:$ & K7-M0 & PMS? & $(\mathrm{N} 95 \mathrm{c})$ \\
\hline RXJ 0333.1+1036 & INT & -0.8 & 0.32 & K3 & PMS & $(\mathrm{N} 95 \mathrm{c})$ \\
\hline RXJ 0336.0+0846 & INT & -0.1 & no & M3 & $\mathrm{dMe}$ & \\
\hline RXJ 0338.1+1224 & ESO & 1.5 & no & K0 & non-PMS & \\
\hline RXJ 0338.3+1020 & INT & 2.0 & 0.25 & G9 & PMS & \\
\hline RXJ 0339.6+0624 & INT & -0.1 & 0.13 & G9 & PMS? & (N95c) \\
\hline RXJ $0340.3+1220$ & INT & -1.1 & no & K5 & $\mathrm{dKe}$ & \\
\hline RXJ 0340.5+0639 & $\mathrm{ESO}$ & 1.6 & no & $\mathrm{K} 2$ & non-PMS & $(3)$ \\
\hline RXJ $0341.2+0453$ & INT & 3.2 & no & G9 & non-PMS & \\
\hline RXJ $0341.2+0759$ & $\mathrm{ESO}$ & 3.2 & no & K0 & non-PMS & \\
\hline RXJ 0343.6+1039 & INT & 1.5 & 0.1 & K0 & PMS? & $\mathrm{SB},(4)$ \\
\hline RXJ 0344.8+0359 & INT & $0.3 \mathrm{f}$ & 0.30 & K3 & PMS? & $(\mathrm{N} 95 \mathrm{c})$ \\
\hline RXJ 0347.2+0933SW & $\mathrm{ESO}$ & -0.40 & 0.4 & K4 & PMS & \\
\hline RXJ $0347.2+0933 \mathrm{NE}$ & ESO & 2.00 & 0.1 & G9 & PMS? & \\
\hline RXJ 0347.9+0616 & INT & 2.6 & 0.2 & G2 & PMS & \\
\hline
\end{tabular}


Table 4. continued

\begin{tabular}{|c|c|c|c|c|c|c|}
\hline designation & tel. & $\begin{array}{r}W_{\lambda}(\mathrm{H} \alpha) \\
(\AA)\end{array}$ & $\begin{array}{r}W_{\lambda}(\mathrm{Li}) \\
(\AA)\end{array}$ & SpType & nature & remarks \\
\hline RXJ 0348.2+1109 & $\mathrm{ESO}$ & 5.6 & no & F5 & non-PMS & HD 23793B \\
\hline RXJ 0348.5+0832 & INT & -0.1 & 0.26 & G7 & PMS & $(5)$ \\
\hline RXJ $0349.4+1255 \mathrm{~N}$ & INT & 2.5 & no & G0 & non-PMS & BD+12 511, $14^{\prime \prime}$ sep. \\
\hline RXJ 0349.4+1255S & INT & 2.0 & no & G7 & non-PMS & $\mathrm{BD}+12$ 511B, $14^{\prime \prime}$ sep. \\
\hline RXJ $0350.2+0849$ & INT & -0.2 & no & K5 & $\mathrm{dKe}$ & $\mathrm{SB}$ \\
\hline RXJ 0350.6+0454 & INT & -0.15 & no & $\mathrm{K} 7$ & $\mathrm{dKe}$ & SB \\
\hline RXJ 0350.6+1033 & $\mathrm{ESO}$ & 2.4 & no & K0 III & non-PMS & \\
\hline RXJ $0351.4+0953 \mathrm{~W}$ & INT & $0.5 \mathrm{f}$ & 0.3 & K1 & PMS? & \\
\hline RXJ 0351.4+0953E & INT & 2.0 & no & F0 & non-PMS & maybe F0 III \\
\hline RXJ $0351.8+0413$ & ESO & 2.00 & 0.12 & G6 & PMS? & $(3),(6)$ \\
\hline RXJ $0352.4+1223$ & ESO & 3.7 & 0.10 & G2 & PMS? & $\mathrm{BD}+11533$ \\
\hline RXJ 0354.1+0528 & $\mathrm{ESO}$ & 2.8 & 0.24 & G8 & PMS & $(3)$ \\
\hline RXJ $0354.3+0535$ & INT & 3.5 & 0.2 & G1 & PMS & $(\mathrm{N} 95 \mathrm{c})$ \\
\hline RXJ 0354.4+1204 & ESO & 3.9 & no & G5 & non-PMS & \\
\hline RXJ $0354.8+1232$ & INT & -1.7 & no & K7 & $\mathrm{dKe}$ & \\
\hline RXJ 0356.7+0943 & INT & -2.7 & no & M3 & $\mathrm{dMe}$ & \\
\hline RXJ $0357.3+1258$ & INT & 1.8 & 0.25 & G0: & PMS & \\
\hline RXJ 0358.1+0932 & INT & $-0.10 \mathrm{f}$ & 0.2 & K3 & PMS? & (N95c) \\
\hline RXJ 0400.0+0730 & INT & 1.3 & no & G3 & non-PMS & \\
\hline RXJ $0400.1+0818 \mathrm{~S}$ & ESO & 2.5 & 0.24 & K0 & PMS? & BD+07 582, $4^{\prime \prime}$ sep. \\
\hline RXJ $0400.1+0818 \mathrm{~N}$ & $\mathrm{ESO}$ & -0.05 & 0.40 & $\mathrm{~K} 2$ & PMS & $\mathrm{BD}+07582 \mathrm{~B}, 4^{\prime \prime}$ sep. \\
\hline RXJ $0400.8+1116$ & INT & 1.8 & no & K0 & non-PMS & \\
\hline RXJ 0402.5+0552 & INT & 1.7 & no & G4 & non-PMS & \\
\hline RXJ 0403.5+0837 & INT & 1.3 & no & K0 & non-PMS & \\
\hline RXJ 0404.4+0519 & INT & $1.0 \mathrm{f}$ & 0.25 & K0 & PMS? & \\
\hline RXJ 0405.5+0324 & INT & -0.4 & no & K4 & $\mathrm{dKe}$ & \\
\hline RXJ $0407.2+0113 \mathrm{~N}$ & INT & 3.3 & 0.2 & G4 & PMS & $2^{\prime \prime}$ sep. \\
\hline RXJ 0407.2+0113S & INT & $0.5 \mathrm{f}$ & 0.35 & K3 & PMS & $2^{\prime \prime}$ sep. \\
\hline RXJ 0407.6+0638 & INT & 2.0 & no & G0 & non-PMS & \\
\hline RXJ 0408.6+1017 & INT & 1.8 & no & G7 & non-PMS & \\
\hline RXJ 0408.8+1028 & $\mathrm{ESO}$ & 3.2 & no & G5 & non-PMS & HD 26172 \\
\hline RXJ 0409.8+1209 & INT & 3.3 & 0.10 & F9 & PMS? & HD 286556 \\
\hline RXJ 0410.6+0608 & INT & -0.05 & 0.11 & K4 & PMS? & $\mathrm{SB} ?$ \\
\hline RXJ 0413.2+1028 & INT & 3.0 & no & G0 & non-PMS & \\
\hline RXJ 0418.6+0143 & INT & $0.4 \mathrm{f}$ & no & K4 & $\mathrm{dKe}$ & \\
\hline RXJ 0419.8+0214 & INT & 4.5 & no & F5 & non-PMS & \\
\hline RXJ 0419.9+0231 & INT & 3.6 & no & F9 & non-PMS & \\
\hline RXJ 0422.9+0141 & INT & 1.5 & yes & F8 & PMS? & $\mathrm{SB},(7)$ \\
\hline RXJ 0423.5+0955 & INT & -0.1 & 0.27 & K4 & PMS? & \\
\hline RXJ 0425.5+1210 & INT & 4.2 & 0.1 & F9 & PMS? & HD 286753 \\
\hline RXJ $0426.4+0957 \mathrm{~W}$ & INT & 7.5 & no & late A & non-PMS & \\
\hline RXJ 0426.4+0957E & INT & 2.9 & 0.14 & G2 & PMS? & \\
\hline RXJ 0427.4+1039 & INT & $1.3 \mathrm{f}$ & 0.35 & G0: & PMS & \\
\hline RXJ 0427.5+0616 & INT & 1.5 & 0.25 & G4 & PMS & \\
\hline RXJ 0427.8+0049 & INT & 3.2 & no & G3 & non-PMS & $\mathrm{BD}+00760, \mathrm{SB}$ \\
\hline RXJ 0429.9+0155 & INT & 1.5 & no & K3 & non-PMS & maybe K3 III \\
\hline RXJ 0433.7+0522 & INT & 4.0 & no & F8 & non-PMS & \\
\hline RXJ $0434.3+0226$ & INT & -0.4 & 0.3 & K4 & PMS & \\
\hline RXJ 0435.5+0455 & INT & 1.2 & no & K3 III & non-PMS & \\
\hline RXJ 0441.9+0537 & INT & abs & no & G5 & non-PMS & $\mathrm{BD}+05706,(8)$ \\
\hline RXJ 0442.3+0118 & INT & -1.0 & no & $\mathrm{K} 2$ & $\mathrm{dKe}$ & \\
\hline RXJ 0442.5+0906 & INT & 1.4 & 0.25 & G7 & PMS & $\mathrm{BD}+08742$ \\
\hline RXJ 0442.6+1018 & INT & 1.2 & no & K3 & non-PMS & maybe K3 III \\
\hline RXJ 0442.9+0400 & INT & 1.1 & 0.22 & K0 & PMS? & \\
\hline
\end{tabular}


Table 4. continued

\begin{tabular}{llrrlll}
\hline designation & tel. & $\begin{array}{r}W_{\lambda}(\mathrm{H} \alpha) \\
(\AA)\end{array}$ & $\begin{array}{r}W_{\lambda}(\mathrm{Li}) \\
(\AA)\end{array}$ & SpType & nature & remarks \\
\hline RXJ 0444.3+0941 & INT & 3.2 & no & F9 & non-PMS & HD 287017, SB \\
RXJ 0444.4+0725 & INT & -0.3 & 0.12 & K5 & PMS? & \\
RXJ 0444.7+0814 & INT & -0.80 & 0.28 & K3 & PMS? & $($ N95c) \\
RXJ 0445.2+0729 & INT & 2.6 & 0.25 & G0 & PMS & \\
RXJ 0445.3+0914 & INT & 3.8 & no & G0 & non-PMS & \\
RXJ 0445.5+1207 & INT & -2.0 & 0.35 & K7 & PMS & \\
RXJ 0448.0+0738 & INT & $-0.1 \mathrm{f}$ & no & K1 & dKe & $($ N95c) \\
RXJ 0450.0+0151 & INT & $0.5 \mathrm{f}$ & 0.35 & K3 & PMS & \\
RXJ 0451.6+0619 & INT & $1.3 \mathrm{f}$ & no & K2 & dKe & SB? \\
RXJ 0459.9+1017 & INT & 3.0 & no & F5 & non-PMS & SB \\
RXJ 0511.2+1031 & INT & -2.8 & 0.65 & K7 & PMS & \\
RXJ 0511.9+1112 & INT & 1.2 & 0.25 & G4 & PMS & \\
RXJ 0512.0+1020 & INT & -0.1 & 0.4 & K2 & PMS & \\
RXJ 0513.6+0955 & INT & 1.4 & no & G6 & non-PMS & \\
RXJ 0515.3+1221 & INT & $1.2 \mathrm{f}$ & no & K0 & dKe & SB, (9) \\
RXJ 0516.3+1148 & INT & $0.1 \mathrm{f}$ & 0.5 & K4 & PMS & $($ N95c) \\
RXJ 0523.0+0934 & INT & 4.2 & no & F8 & non-PMS & \\
RXJ 0523.5+1005 & INT & -0.5 & no & K3 & dKe & SB, (9) \\
RXJ 0523.9+1101 & INT & 3.7 & no & G0 & non-PMS & \\
RXJ 0525.7+1205NW & INT & 1.4 & no & G8 & non-PMS & maybe G8 III \\
RXJ 0525.7+1205SE & INT & 1.4 & no & G8 & non-PMS & maybe G8 III \\
RXJ 0528.4+1213 & INT & 2.5 & no & K2 & non-PMS & \\
RXJ 0528.5+1219 & INT & $0.7 \mathrm{f}$ & 0.35 & K3 & PMS & \\
RXJ 0528.9+1046 & INT & $0.1 \mathrm{f}$ & 0.4 & K3 & PMS & \\
RXJ 0529.3+1210 & INT & -2.0 & 0.35 & K7-M0 & PMS & \\
RXJ 0530.9+1227 & INT & 1.3 & no & K0 & non-PMS & \\
RXJ 0531.8+1218 & INT & -0.74 & 0.5 & K4 & PMS & $($ N95c) \\
\hline
\end{tabular}

Remarks: (1) Spectrum blue-shifted by $2 \AA$; (2) Star A itself is SB, with the secondary having almost the same spectral type as the primary; (3) Also observed at INT; (4) Spectrum blue-shifted by $1 \AA$, the H $\alpha$ line shows a P Cyg profile; (5) H $\alpha$ line shows an inverse P Cyg profile; (6) Spectrum red-shifted by $2 \AA$; (7) SB: the primary is F8 with H $\alpha$ in absorption and $W_{\lambda}(\mathrm{Ca})<W_{\lambda}(\mathrm{Li})=0.16 \AA$, the secondary has $W_{\lambda}(\mathrm{Ca})>W_{\lambda}(\mathrm{Li})=0.13 \AA$, thus the system may by a close PMS binary; H $\alpha$ line shows a P Cyg profile; (8) Very noisy spectrum; (9) Secondary seems to have a slightly earlier spectral type.

\section{Discussion}

From Fig. 1 we see that many of our newly discovered lowmass PMS stars are located up to several tens of degrees away from regions of ongoing star formation. In fact, the area of on-going star formation in Taurus, as defined by the CO contours (Ungerechts \& Thaddeus 1987) and also the area populated by the TTS known before ROSAT or newly discovered by Wichmann et al. (1996), is at $\delta>14^{\circ}$. If the distance of our objects is the same as the TaurusAuriga clouds ( $140 \mathrm{pc})$, these objects will lie up to several tens of parsecs from the clouds. In any case, they are located far from any known star forming region. Despite their location, the lithium equivalent width in most of these stars is indicative of young ages, just typical for wTTS.

Radial velocities for some of them have been presented in Neuhäuser et al. (1995c) and indicate that about half of their 15 objects are kinematic members of the Taurus-
Auriga $\mathrm{T}$ association. A complete analysis of the kinematic status of all stars studied here will be given in Neuhäuser et al. (1997) together with radial velocities (for almost all stars studied here) and proper motions for several stars identified here as new PMS stars.

In any SFR studied the RASS has revealed hundreds of new wTTS, which have been discovered even outside regions of ongoing star formation. However, only few cTTS have been discovered, either by EO or ROSAT. As the RASS is flux-limited and ROSAT pointed observations are spatially biased towards "interesting" regions, many wTTS have not been discovered yet. We find 30 new PMS stars among 115 previously unidentified sources (i.e. 26\%) selected by hardness ratios and $V$ magnitude of the possible counterpart. Although our investigation has been carried out outside molecular gas regions, this percentage of new PMS stars is within the range expected in Neuhäuser et al. (1995a), who predict at least 286 new wTTS among 

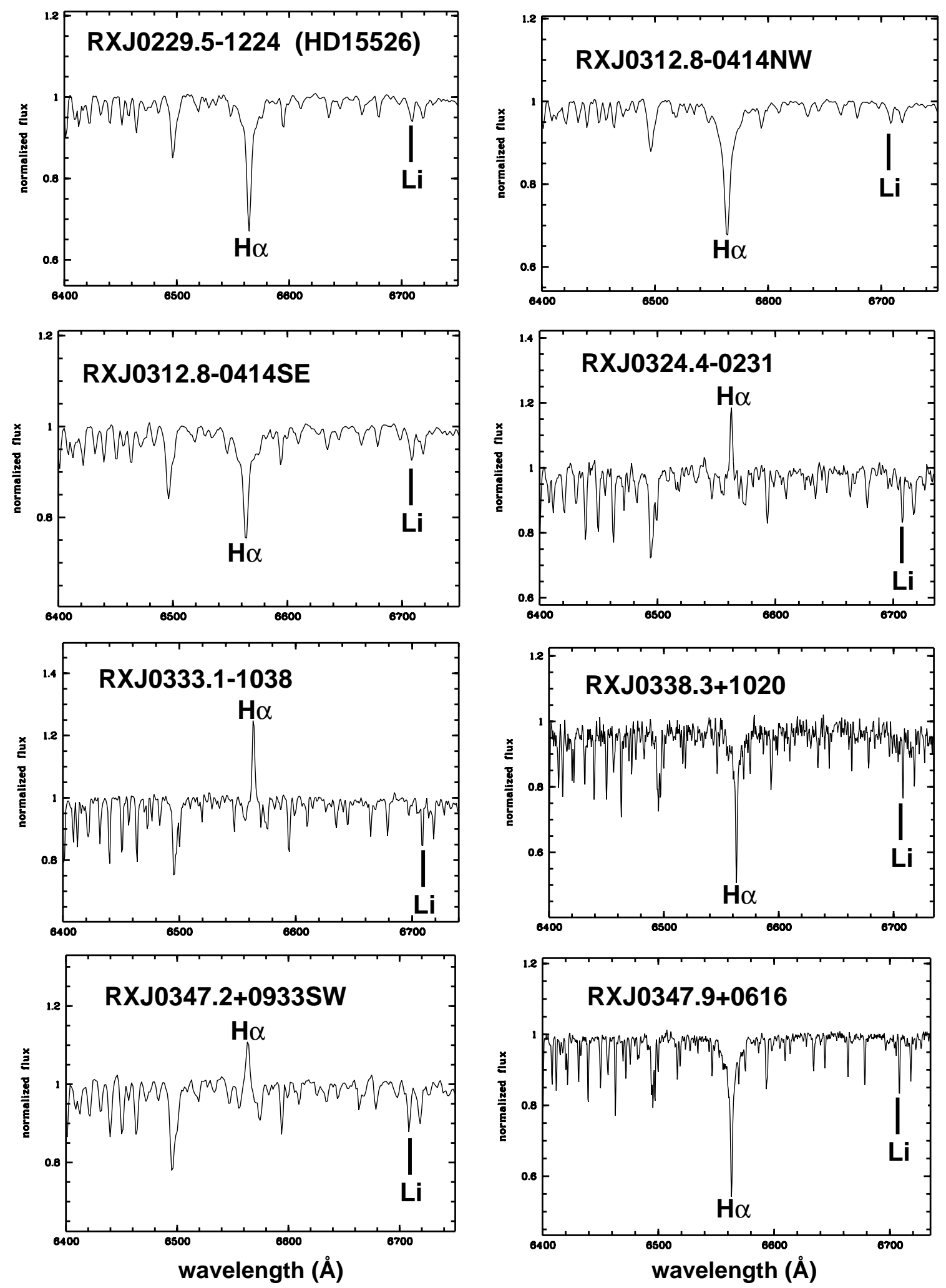

Fig. 5. Spectra of our newly discovered PMS stars 

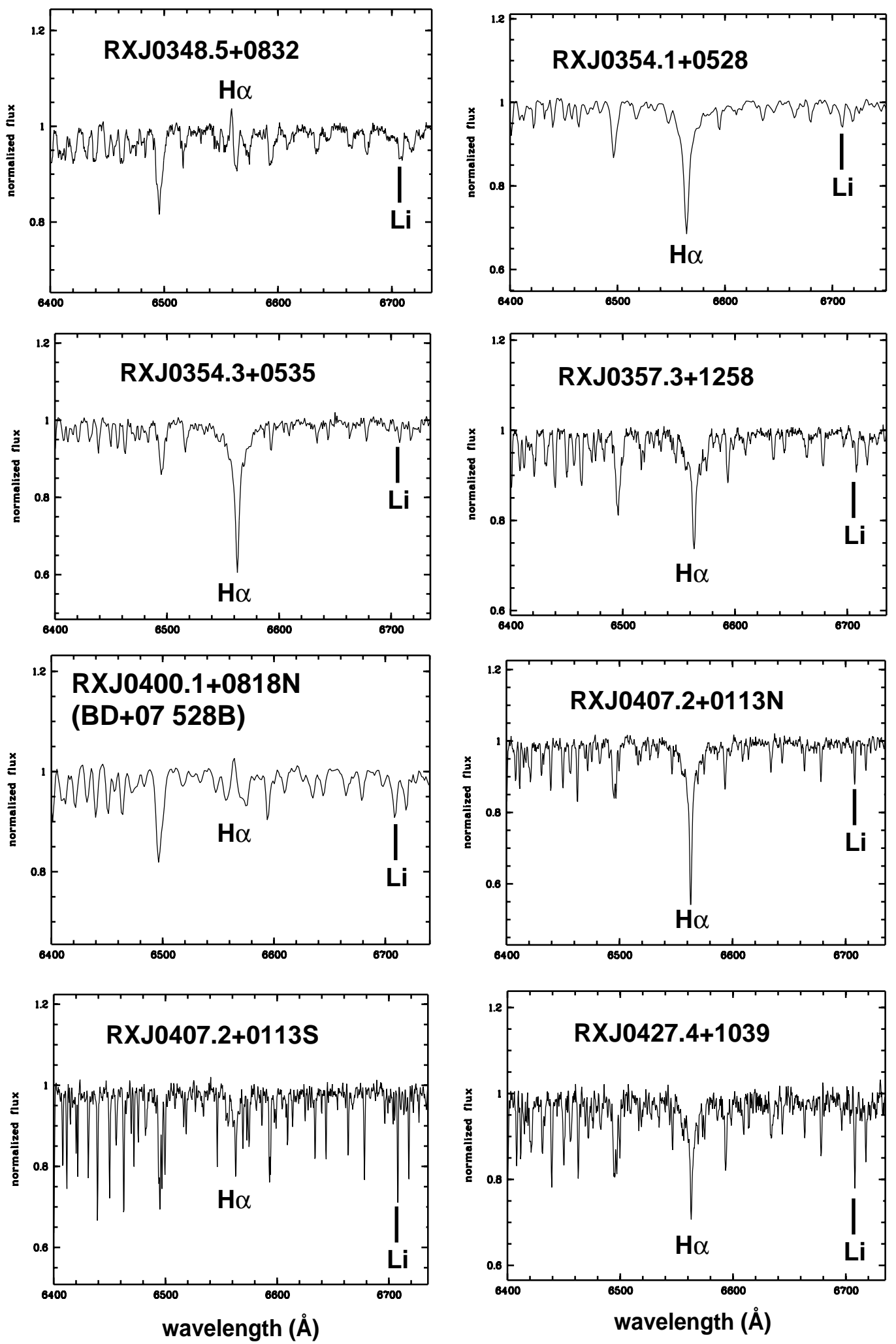

Fig. 5. continued 

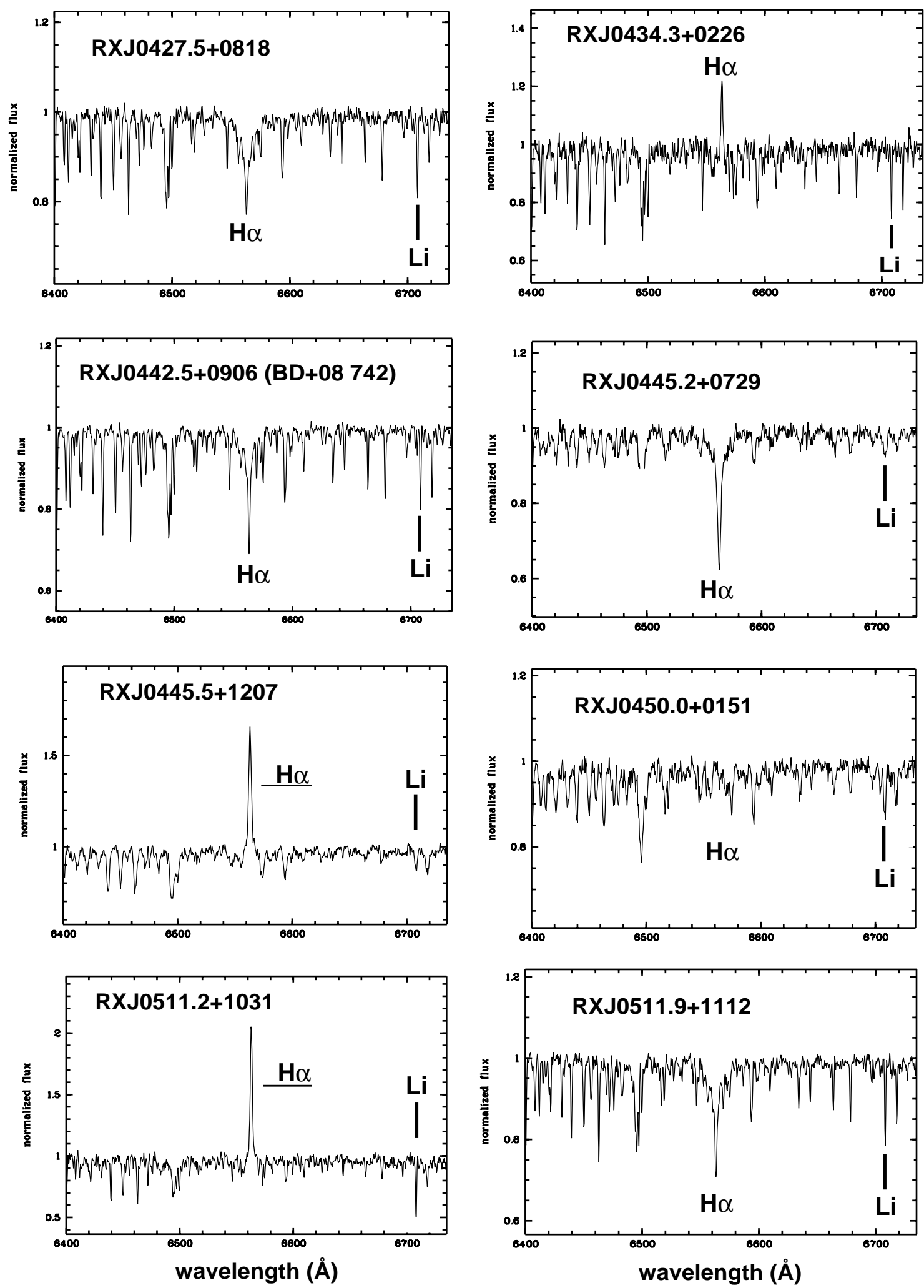

Fig. 5. continued 
as the pre-selection of TTS candidates indicates (e.g. Sterzik et al. 1995). A different explanation has been proposed by Feigelson (1996), who argues that TTS can form in small, high-velocity, short-lived cloudlets within and around a turbulent giant molecular cloud complex (like Taurus-Auriga and Chamaeleon).

Our sample of new low-mass PMS stars found south of the Taurus-Auriga dark cloud complex may contain relatively old dispersed post-TTS, young wTTS formed locally in small short-lived cloudlets, and young wTTS ejected from the central areas of ongoing star formation. With kinematic data (both radial velocities and proper motions) and age estimates (e.g. from placing the stars into the HR-diagram and from precise Li abundances) one may be able to distinguish between these different contributions in the future. The presence of lithium in a large number of stars in the general direction of any known SFR studied is an observational fact that certainly needs further explanation.

Acknowledgements. We would like to thank Guillermo Torres for fruitful discussions. Many thanks also to the EXSAS and ROSAT teams at MPE as well as to the support teams at the different telescope sites used in this research. This work has made use of the SIMBAD database operated at CDS, Strasbourg. The ROSAT project has been supported by the Bundesministerium für Bildung, Wissenschaft, Forschung und Technologie (BMBW/DARA) and the MaxPlanck-Gesellschaft.

\section{References}

Alcalá J.M., Krautter J., Schmitt J.H.M.M., et al., 1995, A\&AS 114, 109

Bastian U., Finkenzeller U., Jascheck C., Jascheck M., 1983, A\&A 126, 438

Carkner L., Feigelson E.D., Koyama K., Montmerle T., Reid N., 1996, ApJ 464, 286

Elias J.H., 1978, ApJ 224, 857

Feigelson E.D., 1996, ApJ 468, 306

Feigelson E.D., DeCampli W.M., 1981, ApJ 243, L89

Gahm G.F., 1980, ApJ 242, L163

García López R.J., Rebolo R., Martín E.L., 1994, A\&A 282, 518

Herbig G.H., 1962, Adv. Astron. Astrophys. 1, 47

Herbig G.H., 1978, in: Mirzoyan L. (ed.) Problems of Physics and Evolution of the Universe. Academy of Science of Armenia, Erevan, p. 171

Herbig G.H., Bell K.R., 1988, Lick Observatory Bulletin No. 1111

Jacoby G.H., Hunter D.A., Christian C.A., 1984, ApJS 56, 257

Jones B.F., Herbig G.H., 1979, AJ 84, 1872

Kenyon S.J., Dobrzycka D., Hartmann L.W., 1994, AJ 108, 1872

Krautter J., 1996, The impact of ROSAT observations on our understanding of star forming regions. In: Pallavicini R., Dupree A. (eds.) Proc. of 9th Cambridge Workshop on Cool Stars, Stellar Systems, and the Sun, p. 395

Lindroos K.P., 1986, A\&A 156, 223

Magazzù A., Rebolo R., Pavlenko Ya.V., 1992, ApJ 392, 159

Martín E.L., Magazzù A., Rebolo R., 1992, A\&A 257, 186

Martín E.L., Magazzù A., Rebolo R., Pavlenko Ya.V., 1994, A\&A 282, 503

Neuhäuser R., 1996, in: Zimmermann H.U., Trümper J.E., Yorke H. (eds.) Röntgenstrahlung from the Universe. MPE Report 263, Conf. Proc., p. 13

Neuhäuser R., Sterzik M.F., Schmitt J.H.M.M., Wichmann R., Krautter J., 1995a, A\&A 295, L5

Neuhäuser R., Sterzik M.F., Schmitt J.H.M.M., Wichmann R., Krautter J., 1995b, A\&A 297, 391

Neuhäuser R., Sterzik M.F., Torres G., Martín E.L., 1995c, A\&A 299, L13

Neuhäuser R., Torres G., Sterzik M.F., Randich S., 1997, A\&A (in press)

Pallavicini R., Pasquini L., Randich S., 1992, A\&A 261, 245

Pfeffermann E., Briel U.G., Hippmann H., et al., 1988, in: Proc. SPIE 733, p. 519

Raymond J.C., Smith B.S., 1977, ApJS 35, 419

Schmitt J.H.M.M., Zinnecker H., Cruddace R., Harnden F.R., 1993, ApJ 402, L13

Soderblom D.R., Jones B.F., Balachandran S., et al., 1993, AJ 106, 1059

Sterzik M.F., Alcalá J.M., Neuhäuser R., Schmitt J.H.M.M., 1995, A\&A 297, 419

Sterzik M.F., Durisen R., 1995, A\&A 304, L9

Strom K.M., Strom S.E., 1994, ApJ 424, 237

Trümper J.E., 1983, Adv. Space Res. 2, 241

Ungerechts H., Thaddeus P., 1987, ApJS 63, 645

Walter F.M., 1986, ApJ 306, 573

Walter F.M., Brown A., Mathieu R.D., Myers P.C., Vrba F.J., 1988, AJ 96, 297

Wichmann R., Krautter J., Schmitt J.H.M.M., et al., 1996, A\&A 312,439 